\title{
Article \\ Changes in Glycated Human Serum Albumin Binding Affinity for Losartan in the Presence of Fatty Acids In Vitro Spectroscopic Analysis
}

\author{
Agnieszka Szkudlarek*(D), Jadwiga Pożycka, Karolina Kulig, Aleksandra Owczarzy, Wojciech Rogóż and \\ Małgorzata Maciążek-Jurczyk (iD)
}

check for updates

Citation: Szkudlarek, A.; Pożycka, J.; Kulig, K.; Owczarzy, A.; Rogóż, W.; Maciążek-Jurczyk, M. Changes in Glycated Human Serum Albumin Binding Affinity for Losartan in the Presence of Fatty Acids In Vitro Spectroscopic Analysis. Molecules 2022, 27, 401. https://doi.org/ $10.3390 /$ molecules27020401

Academic Editor: Igor Ivanov

Received: 3 December 2021

Accepted: 6 January 2022

Published: 8 January 2022

Publisher's Note: MDPI stays neutral with regard to jurisdictional claims in published maps and institutional affiliations.

Copyright: (C) 2022 by the authors. Licensee MDPI, Basel, Switzerland. This article is an open access article distributed under the terms and conditions of the Creative Commons Attribution (CC BY) license (https:// creativecommons.org/licenses/by/ $4.0 /)$.
Department of Physical Pharmacy, Faculty of Pharmaceutical Sciences in Sosnowiec, Medical University of Silesia in Katowice, 40-055 Katowice, Poland; jpozycka@sum.edu.pl (J.P.); kkulig@sum.edu.pl (K.K.); aowczarzy@sum.edu.pl (A.O.); wrogoz@sum.edu.pl (W.R.); mmaciazek@sum.edu.pl (M.M.-J.)

* Correspondence: aszkudlarek@sum.edu.pl; Tel.: +48-32-364-1581

\begin{abstract}
Conformational changes in human serum albumin due to numerous modifications that affect its stability and biological activity should be constantly monitored, especially in elderly patients and those suffering from chronic diseases (which include diabetes, obesity, and hypertension). The main goal of this study was to evaluate the effect of a mixture of fatty acids (FA) on the affinity of losar$\tan$ (LOS, an angiotensin II receptor $\left(\mathrm{AT}_{1}\right)$ blocker used in hypertension, a first-line treatment with coexisting diabetes) for glycated albumin—simulating the state of diabetes in the body. Individual fatty acid mixtures corresponded to the FA content in the physiological state and in various clinical states proceeding with increased concentrations of saturated (FAS) and unsaturated (FAUS) acids. Based on fluorescence studies, we conclude that LOS interacts with glycated human serum albumin (af)gHSA in the absence and in the presence of fatty acids ((af)gHSA phys, (af)gHSA 4 ,,$($ af $) g H_{S A}$, (af)gHSA 4 US, and (af)gHSA 8 US $)$ and quenches the albumin fluorescence intensity via a static quenching mechanism. LOS not only binds to its specific binding sites in albumins but also non-specifically interacts with the hydrophobic fragments of its surface. Incorrect contents of fatty acids in the body affect the drug pharmacokinetics. A higher concentration of both FA and FA $A_{U}$ acids in glycated albumin reduces the stability of the complex formed with losartan. The systematic study of FA and albumin interactions using an experimental model mimicking pathological conditions in the body may result in new tools for personalized pharmacotherapy.
\end{abstract}

Keywords: spectroscopic methods; glycated human serum albumin; saturated and unsaturated fatty acids; losartan

\section{Introduction}

Human serum albumin (HSA), being the main protein in plasma, is essential in many processes taking part in the body. HSA performs key functions in maintaining homeostasis in the body, e.g., HSA controls the plasma oncotic pressure, modulates the fluid distribution between the body compartments, displays antioxidant and enzymatic properties, and inactivates toxic compounds [1-4]. HSA has the ability to transport many biologically active compounds through binding endo- and the exogenous compounds (e.g., fatty acids, metal ions, drugs, hormones, vitamins, toxins, and metabolites) [1,4].

One of the processes causing the loss of albumins original properties is the increased glycation in a state of hyperglycemia. Heterogeneous, stable compounds formed at the end of this process-Advanced Glycation End-Products (AGEs) - play a significant role in the development of chronic micro- and macroangiopathic diabetic complications as well as degenerative processes related to age $[5,6]$. 
Fatty Acids (FAs) perform many important function in living organisms, e.g., they are used as energy substrates in the $\beta$-oxidation process; as a building material for phospholipids, which are, in turn, used to create biological membranes; and they are precursors of important biological mediators, such as prostaglandins, leukotrienes, and thromboxanes $[4,7]$.

FAs are also involved in intracellular transmission and take part in post-transcriptional modification processes [7]. As components of complex lipids, FAs play an important role in the electric and thermal isolation of the body, as well as provide it with mechanical protection. Due to the fatty acid low solubility in the blood plasma, albumin is the main transporter of FA [8]. The albumin-FA complex is in equilibrium with a very small fraction of unresolved FA dissolved in the plasma (less than $0.01 \%$ of the total pool).

Apart from the two main versatile ligand binding sites with a high affinity for diverse molecules referred to as 'Sudlow's sites' — site I (located in subdomain IIIA) and site II (in subdomain IIA) [9] - there are nine fatty acid binding sites in the HSA molecule (i.e., FA1-FA9), which are arranged in an asymmetrical manner and include all six subdomains [10] (Figure 1).

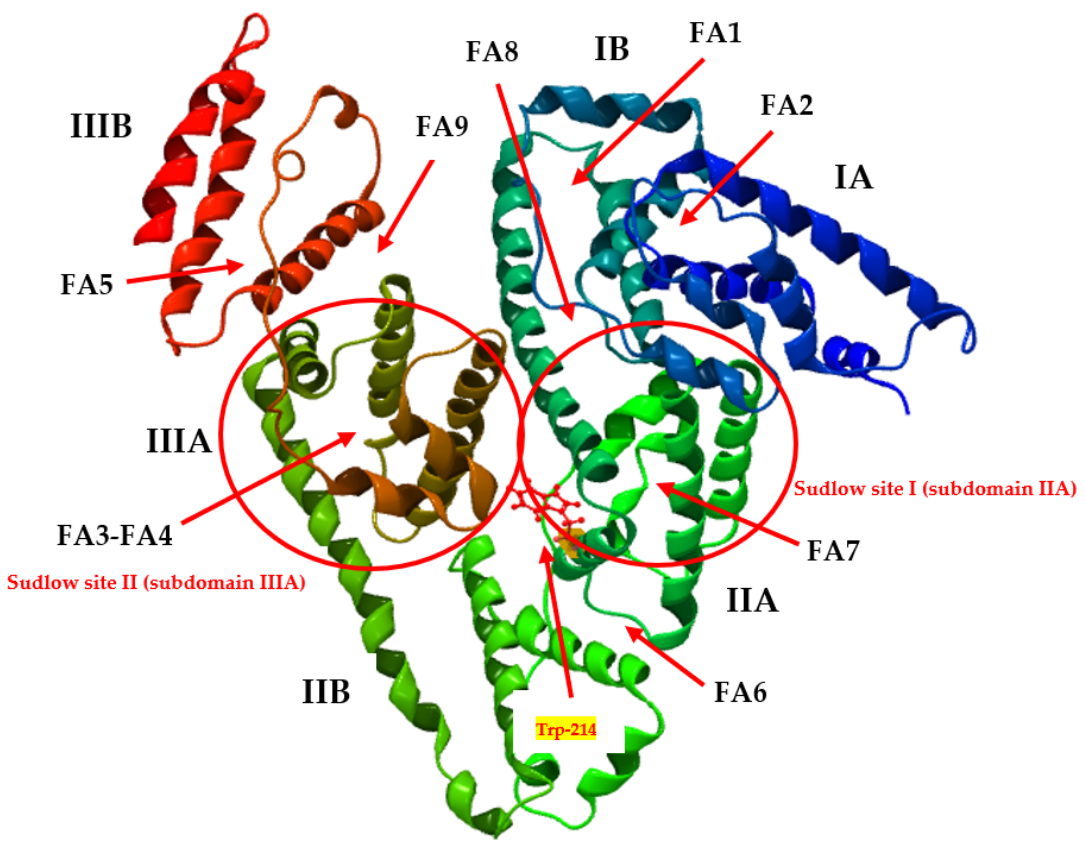

Figure 1. Human serum albumin (HSA) drug binding sites (Sudlow site I and II) with the location of main fatty acids in the HSA molecule (FA1-FA9) and the marked Trp-214 residue. Molecular graphic image based on [11] was produced using the CLC Drug Discovery Workbench version 1.0.2. (CLC Bio, a QIAGEN Company: Aarhus, Denmark) [license number: CLC-LICENSE-51JT8-DXYBY2A3EW-ED80P-DGW80] (PDB ID: 4K2C).

On one side, the non-polar bonds with fatty acids protect the tertiary structure of albumin against denaturation with guanidine hydrochloride, urea, and temperature. On the other side, there is a conformational change in the macromolecule and an increase in Cys-34 reactivity as a result of the exposure of the sulfhydryl residue [12]. Structural changes in Sudlow's site I concern the reorganization of hydrogen bonds between amino acid residues Tyr-150, Glu-153, Gln-196, His-242, Arg-257, and His-288, which leads to an increase in the area of the binding pocket and a polarity disorder.

In Sudlow's site II, there is a change in the conformation of the Leu-387 and Leu-453, and the breaking of Arg-348 and Glu-450 bonds. This allows the fatty acids, bound at FA3, to gain access to the polar region around FA4 [12]. Unmodified human serum albumin binds anions, when in combination with fatty acids, indicates an increased affinity for substances in the form of cations [13]. 
Many experiments have shown that the presence of fatty acids can have a significant impact on the process of drugs binding by albumin, particularly drugs with a high affinity for macromolecules. Fatty acids can compete for the HSA molecule binding sites or cooperate with drugs, wherein the FA affinity for albumin decreases with every filled macromolecule binding site $[14,15]$.

It is important as ligands bound to one binding site can change the structure or number of other binding sites in the albumin molecule [12]. Due to the diversity present in the results concerning change in pharmacological action through research revolving around in vitro and in vivo studies of exogenous ligand interactions, the binding mechanisms of drugs in the presence of fatty acids with a transport protein requires thorough study.

The increasing occurrence of obesity related to, i.a., the overuse of saturated fatty acids in the diet is a predisposing factor to the appearance of metabolic syndrome, which significantly increases the risk of type 2 diabetes and cardiovascular disease in adults [16]. Its main components, apart from obesity, are primarily arterial hypertension, insulin resistance, and atherogenic dyslipidemia. Losartan (LOS, Figure 2) is one of the significant drugs used in the regulation of arterial hypertension as well as in the treatment for chronic heart failure and the prevention of cardiovascular diseases in order to reduce the risk of a stroke in patients with hypertension and left ventricular hypertrophy. As a selective and competitive, nonpeptide angiotensin II (AII) receptor antagonist, LOS blocks the vasoconstrictor and aldosterone-secreting effects of angiotensin II [17].

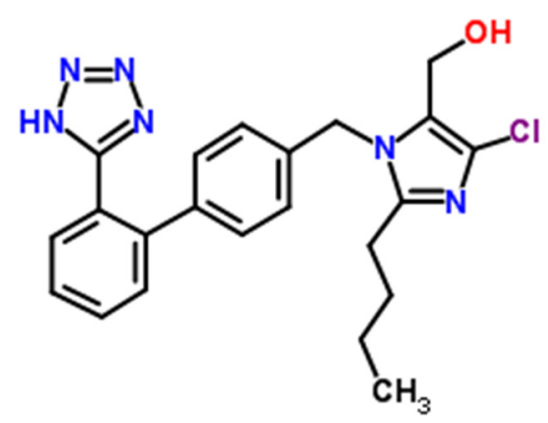

Figure 2. The chemical structure of losartan ((2-Butyl-4-chloro-1-\{[2'-(1H-tetrazol-5-yl)-4biphenylyl]methyl $\}-1 H$-imidazol-5-yl)methanol, LOS). The structural formula of losartan was drawn with the use of the ACD/ChemSketch ver. 2018.2.1 program.

The aim of this study was to evaluate the effect of fatty acid (FA) mixtures with different saturated (PA - palmitic acid, MYR - myristic acid, and SA — stearic acid) and unsaturated (OA-oleic acid and LA - linoleic acid) fatty acids on the affinity of losartan for glycated human serum albumin-simulating the state of diabetes in the body. Individual fatty acid mixtures corresponded to the FA content in physiological state and in various clinical states proceeding with increased concentrations of saturated (FAS) and unsaturated (FAUS) acids. The binding properties of glycated albumin in the presence fatty acids and conformational changes of glycated human serum albumin were studied based on the quantitative analysis using absorption (UV-Vis) and fluorescence spectroscopy.

As has been well described in the literature, both UV-Vis and fluorescence spectroscopy, mainly the quenching of biomacromolecules fluorescence method, are very helpful in protein-ligand and protein-ligand-ligand interactions due to their high sensitivity, rapidity, and ease of implementation [18-22]. Fluorescence measurements can provide some information about the binding of small molecules to proteins, such as the binding mechanism, binding mode, binding constants, and binding sites, and are useful in drug development in the early stage of research [19].

The research regarding the influence of fatty acids on the structure and binding properties of glycated human albumin, which simulates the states of diabetes in the body, is important from the scientific point of view because the conformational transformation of the most important transport protein—serum albumin—due to the many modifications 
that affect its stability and biological activity. This protein should be constantly monitored, especially in diseases and in the elderly. Monitoring the concentration of the drug-free fraction can help with optimizing pharmacotherapy as well as increase its effectiveness and avoid side effects.

\section{Results and Discussion}

2.1. The Interaction of Losartan with Glycated Human Serum Albumin in the Absence and in the Presence of Fatty Acids

Based on the emission fluorescence spectra of glycated, defatted (af)gHSA and glycated in the presence of fatty acids (af)gHSA phys, (af)gHSA 4 , (af)gHSA 8 , (af)gHSA 4 US, and (af)gHSA ${ }_{8 \mathrm{US}}$ albumin $\left(5 \times 10^{-6} \mathrm{~mol} \cdot \mathrm{L}^{-1}\right)$ (data not shown), an increase in the losartan (LOS) concentration $\left(5 \times 10^{-6} \mathrm{~mol} \cdot \mathrm{L}^{-1}-5 \times 10^{-5} \mathrm{~mol} \cdot \mathrm{L}^{-1}\right)$ in ligand-albumin systems causes a gradual decrease in the macromolecule fluorescence intensity. According to Stryer theory, the observed effect may be associated with the quenching fluorescence of excited fluorophores (tryptophanyl residue (Trp-214) and tyrosyl residues (Tyrs)) of glycated human albumins by the losartan molecule, which was found in no more than $10 \mathrm{~nm}$ proximity [18].

This distance makes it possible to transfer energy to the ligand molecule. In addition, in the LOS-(af)gHSA system (from a 0:1 to 10:1 molar ratio), after excitation at $\lambda_{\mathrm{ex}}=275 \mathrm{~nm}$ and $\lambda_{\text {ex }}=295 \mathrm{~nm}$, the shift in the defatted albumin fluorescence emission band towards shorter waves (blue shift) by $13 \mathrm{~nm}\left(\Delta \lambda_{\max }=326-313 \mathrm{~nm}\right)$ and $2 \mathrm{~nm}\left(\Delta \lambda_{\max }=337-335 \mathrm{~nm}\right)$ relative to the spectrum of the ligand-free albumin has been observed. The hypsochromic shift of maximum albumin fluorescence indicates the formation of a hydrophobic environment around the tryptophanyl (Trp-214) and residues tyrosyl (Tyrs) of (af)gHSA due to the interaction of LOS with albumin.

The shift in the fluorescence emission band of albumin relative to the albumin spectrum in the presence of LOS $\left(\Delta \lambda_{\max }\right)$ smaller for LOS-(af)gHSA phys $\left(\Delta \lambda_{\max }=324-313 \mathrm{~nm}\right)$, LOS-(af)gHSA 4 S $\left(\Delta \lambda_{\max }=324-316 \mathrm{~nm}\right)$, LOS-(af)gHSA 8 $\left(\Delta \lambda_{\max }=323-318 \mathrm{~nm}\right)$, LOS(af)gHSA 4 US $\left(\Delta \lambda_{\max }=323-318 \mathrm{~nm}\right)$, and LOS-(af)gHSA $8 \mathrm{US}\left(\Delta \lambda_{\max }=322-320 \mathrm{~nm}\right)$ systems than for the LOS-(af)gHSA system at the excitation wavelength $\lambda_{\mathrm{ex}}=275 \mathrm{~nm}$, may indicate less variability of the hydrophobic/hydrophilic properties of LOS binding site as a result of the content of fatty acids in the structure of albumin (near the Trp-214 residue in subdomain IIA and Tyrs residues in subdomains IB, IIB, IIA, and IIIA).

At the excitation $\lambda_{\mathrm{ex}}=295 \mathrm{~nm}$, no shift has been recorded. Lakowicz explained that the emission of indole Trp-214 may be blue shifted if the group is buried within a native protein, and its emission may shift to longer wavelengths (red shift) when protein is unfolded [19]. Similarly, as in our previous work, the presence of acetohexamide (AH) - a drug with hypoglycemic activity and a sulfonylurea derivative of the first generation-also caused a blue shift of glycated human serum albumin in the absence of FA (af)gHSA spectra in AH-(af)gHSA [23].

The blue shift of maximum albumin fluorescence $\left(\Delta \lambda_{\max }\right)$ caused by the presence of losartan indicates the possibility of hydrophobic interactions between the aromatic rings of the LOS molecule and aromatic amino acid rings of the hydrophobic albumins cavity within IIA (Trp-214, Tyr-263) or/and IB (Tyr-138, Tyr-140, Tyr-148, Tyr-150, andTyr-161), IIB (Tyr-319, Tyr-332, Tyr-334, Tyr-341, Tyr-353, and Tyr-370) and IIIA (Tyr-401, Tyr-411, Tyr-452, and Tyr-497) subdomains $[19,24]$.

Moeinpour et al., using a molecular dynamics simulation technique, also studied the interaction between losartan and glycated human serum albumin (gHSA). Based on the results visualized by Ligplus and Autodock (Figure 11 from [25]), they concluded that LOS was located within the hydrophobic binding pocket of gHSA, and several phenyl groups of the drug interacted with the Glu-348, Glu-345, Val-346, Lys-373, Leu-369, Phe-349, Lys-364, Asp-372, Glu-360, and Asn-361 residues of subdomain IIB of gHSA through hydrophobic interaction. 
Contrary to the study of LOS interaction with HSA, the specific hydrogen bonding interaction observed between the NH group of LOS and Asn-391 residue of albumin has not been identified. The environment of subdomain IIB, fatty acid high- (FA4), and low(FA3, FA6, and FA7) affinity binding site, is likely the place where losartan can be located, and these sites could affect the binding.

Moreover, by the use of multiple spectroscopic methods, Moeinpour et al. also observed a blue shift of HSA maximum wavelength (fatty-acid-free human serum albumin), as well as its glycated form (gHSA) with an increasing amount of losartan [25]. This effect explained that the chromophore of HSA and gHSA was found to be directed towards more hydrophobic environments, and the conformation of the proteins was changed by the presence of the drug.

Fluorescence quenching curves present $5 \times 10^{-6} \mathrm{~mol} \cdot \mathrm{L}^{-1}$ glycated human serum albumin (af)gHSA in the absence and in the presence of fatty acids ((af)gHSA ${ }_{\text {phys }}$ (af)gHSA 4 , (af) $\mathrm{gHSA}_{8 \mathrm{~S}}$, (af)gHSA${ }_{4 \mathrm{US}}$, and (af)gHSA $8 \mathrm{US}$ ) fluorescence quotient in the absence $\left(\mathrm{F}_{0}\right)$ and in the presence of LOS $\left(5 \times 10^{-6} \mathrm{~mol} \cdot \mathrm{L}^{-1}-5 \times 10^{-5} \mathrm{~mol} \cdot \mathrm{L}^{-1}\right)(\mathrm{F})$ in the function of the drug:albumin molar ratio, $\lambda_{\mathrm{ex}}=275 \mathrm{~nm}$ and $\lambda_{\mathrm{ex}}=295 \mathrm{~nm}$ (Figure 3).

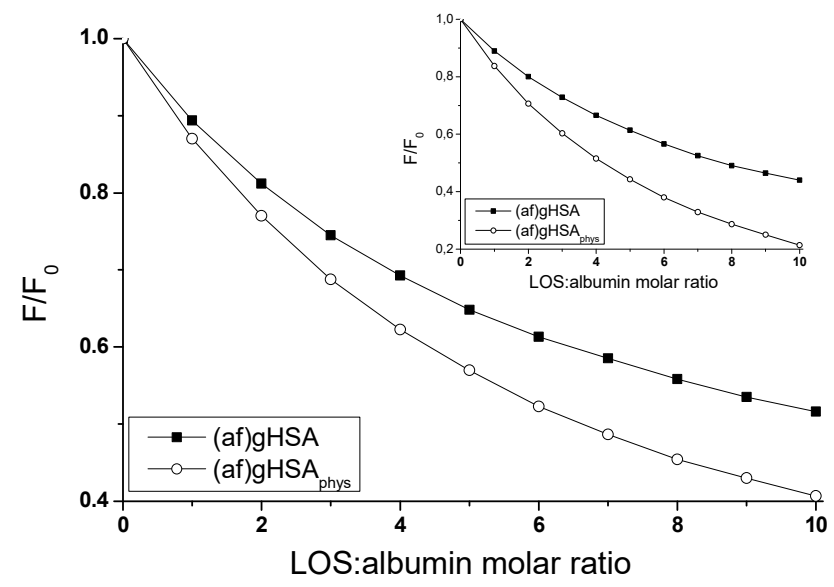

(a)

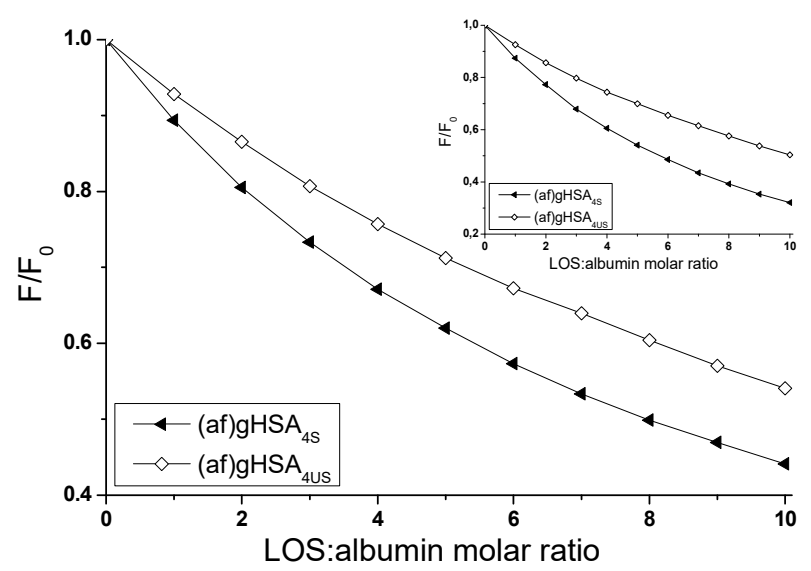

(b)

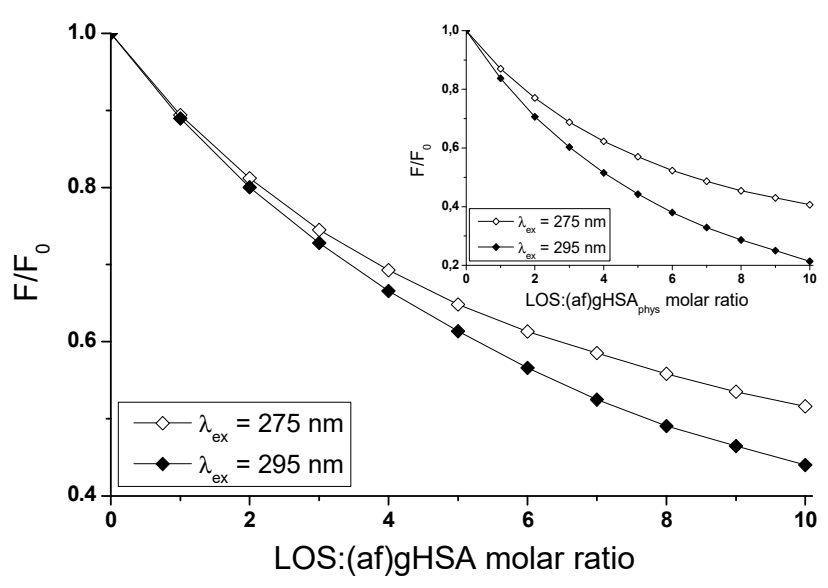

(d)

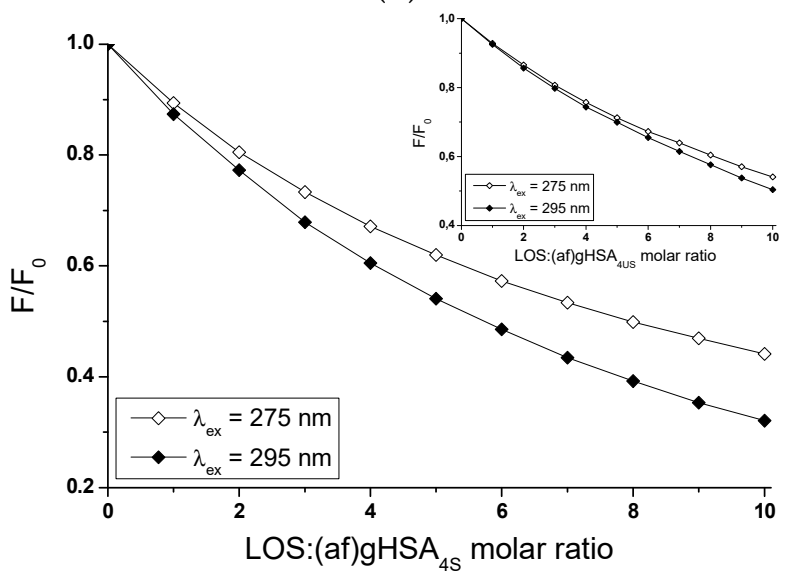

(e)

Figure 3. Cont. 


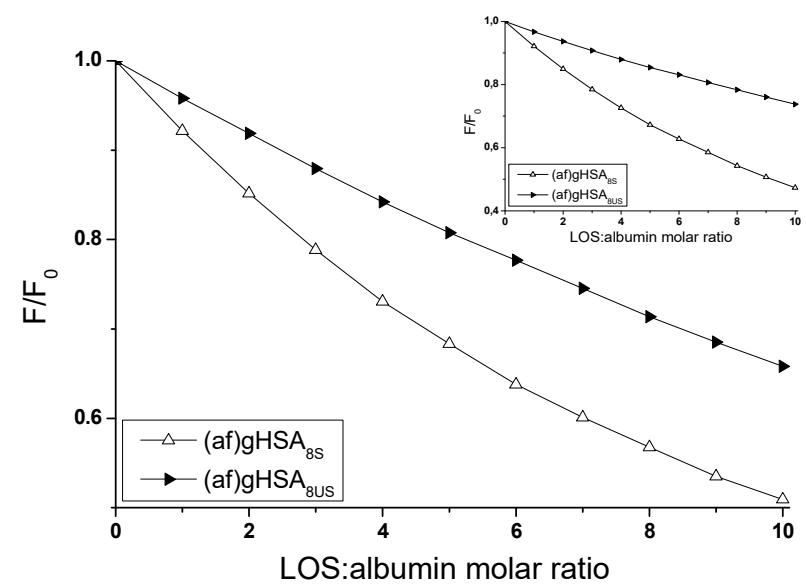

(c)

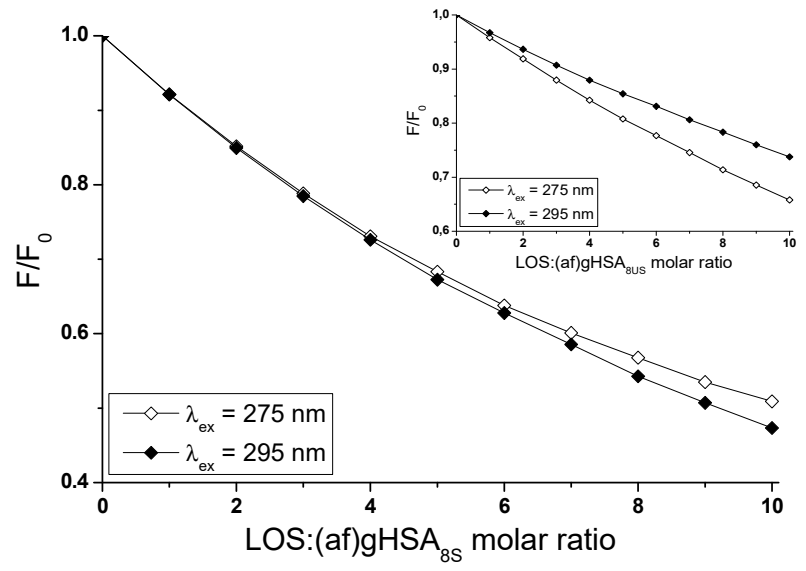

(f)

Figure 3. Fluorescence quenching of (a) (af)gHSA, (af)gHSA phys, (b) (af)gHSA 4 , (af)gHSA 4 US, (c) (af)gHSA 8 , (af)gHSA 8 US complexed with LOS $\left(5 \times 10^{-6} \mathrm{~mol} \cdot \mathrm{L}^{-1}-5 \times 10^{-5} \mathrm{~mol} \cdot \mathrm{L}^{-1}\right)$, $\lambda_{\mathrm{ex}}=275 \mathrm{~nm}$ (in the main view), $\lambda_{\mathrm{ex}}=295 \mathrm{~nm}$ (in the insert) and fluorescence quenching of (d) LOS-(af)gHSA, (e) LOS-(af)gHSA 4 , (f) LOS-(af)gHSA 8 system (in the main view) and (d) LOS(af)gHSA phys $_{\text {, }}$ (e) LOS-(af)gHSA 4 US, and (f) LOS-(af)gHSA 8 US system (in the insert) for $\lambda_{\mathrm{ex}}=275 \mathrm{~nm}$ and $\lambda_{\mathrm{ex}}=295 \mathrm{~nm}$; the albumin concentration was $5 \times 10^{-6} \mathrm{~mol} \cdot \mathrm{L}^{-1}$; the error bars are smaller than the symbols.

The course of albumin fluorescence quenching curves illustrates the reduction in fluorescence intensity of human serum albumin (af)gHSA in the absence of fatty acids and with fatty acids ((af)gHSA ${ }_{\text {phys }}$, (af)gHSA 4 , (af)gHSA 8 , (af)gHSA 4 US, and (af)gHSA 8 Us)) with the increase of losartan concentration in LOS-glycated albumin system (Figure 3a-C, in the main view and in the insert). The presence of fatty acids affects the ability of losartan to quench albumin fluorescence.

Table 1 shows the percentage of fluorescence quenching (af)gHSA and (af)gHSA phys' (af)gHSA 4 and (af)gHSA 4 US, (af)gHSA 8 and (af)gHSA 8 US $\left(5 \times 10^{-6} \mathrm{~mol} \cdot \mathrm{L}^{-1}\right)$ for the highest concentration of LOS $\left(5 \times 10^{-5} \mathrm{~mol} \cdot \mathrm{L}^{-1}\right)$. The data collected in Table 1 show that the strongest quenching of albumin fluorescence in the presence of losartan with the increase of concentration was in the range of $59.34 \%$ and $77.80 \%$ for (af)gHSA $\mathrm{phys}_{\text {s }}$ at $\lambda_{\mathrm{ex}}=275 \mathrm{~nm}$ and $\lambda_{\mathrm{ex}}=295 \mathrm{~nm}$, respectively.

Table 1. Fluorescence quenching of LOS-(af)gHSA, LOS-(af)gHSA ${ }_{\text {phys }}$, LOS-(af)gHSA 4 , LOS(af)gHSA ${ }_{4 U S}$, LOS-(af)gHSA 8 , an LOS-(af)gHSA 8 Us systems and the fluorescence quenching percentage at $\lambda_{\mathrm{ex}}=275 \mathrm{~nm}$ and $\lambda_{\mathrm{ex}}=295 \mathrm{~nm}$ excitation wavelength; albumin and losartan concentrations were $5 \times 10^{-6} \mathrm{~mol} \cdot \mathrm{L}^{-1}$ and $5 \times 10^{-5} \mathrm{~mol} \cdot \mathrm{L}^{-1}$, respectively.

\begin{tabular}{|c|c|c|c|}
\hline \multirow{2}{*}{$\begin{array}{c}\text { Ligand-Albumin } \\
\text { System }\end{array}$} & $\lambda_{\mathrm{ex}}=275 \mathrm{~nm}$ & $\lambda_{\mathrm{ex}}=295 \mathrm{~nm}$ & \multirow{2}{*}{$\begin{array}{c}\text { Difference between } \\
\lambda_{\mathrm{ex}}=275 \mathrm{~nm} \text { and } \lambda_{\mathrm{ex}}=295 \mathrm{~nm}\end{array}$} \\
\hline & \multicolumn{2}{|c|}{ Fluorescence Quenching Percentage (\%) } & \\
\hline LOS-(af)gHSA & $48.36 \%$ & $55.99 \%$ & $7.63 \%$ \\
\hline LOS-(af)gHSA ${ }_{\text {phys }}$ & $59.34 \%$ & $77.80 \%$ & $18.46 \%$ \\
\hline LOS-(af)gHSA & $55.89 \%$ & $67.93 \%$ & $12.04 \%$ \\
\hline LOS-(af)gHSA 85 & $49.08 \%$ & $52.66 \%$ & $3.58 \%$ \\
\hline LOS-(af)gHSA 4 US & $45.91 \%$ & $49.63 \%$ & $3.72 \%$ \\
\hline LOS-(af)gHSA 8 US & $34.20 \%$ & $26.23 \%$ & $7.97 \%$ \\
\hline
\end{tabular}

This means that losartan is sufficiently close to protein tryptophanyl or/and tyrosyl residues (not more than $10 \mathrm{~nm}$ ) and has the strongest affinity for (af)gHSA phys molecule than for (af)gHSA and (af)gHSA 4 , (af)gHSA 8 , (af)gHSA 4 Us, and (af)gHSA 8 Us. A stronger fluorescence quenching for (af)gHSA $\mathrm{phys}_{\text {s }}$ than (af)gHSA (Figure 3a), for (af)gHSA 4 s than (af)gHSA 4 Us (Figure $3 \mathrm{~b}$ ), for (af)gHSA 8 s than (af)gHSA 8 Us (Figure 3c), at both excitation 
wavelengths $\lambda_{e x}=275 \mathrm{~nm}$ (in the main view) and $\lambda_{\mathrm{ex}}=295 \mathrm{~nm}$ (in the insert), has been observed.

This demonstrates a higher losartan ability to absorb energy from excited fluorophores of albumin in the presence of fatty acids at physiological concentration ( (af)gHSA $\left.\mathrm{phys}_{\text {s }}\right)$ than from defatted albumin ((af)gHSA) and from albumin containing four times ((af)gHSA $\mathrm{gS}_{4 \mathrm{~S}}$ and eight-times ((af)gHSA 8 S $)$ higher amounts of saturated than unsaturated ((af)gHSA 4 US,

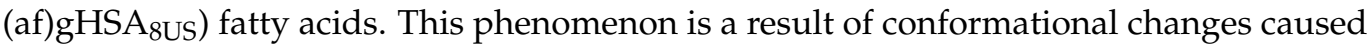
by the presence of fatty acids at physiological concentration or lower contents of saturated and unsaturated fatty acids.

As previously mentioned, after the excitation of albumin at $\lambda_{\mathrm{ex}}=295 \mathrm{~nm}$, the observed emission of fluorescence comes almost exclusively from a tryptophanyl residue (Trp-214), while, for $\lambda_{\mathrm{ex}}=275 \mathrm{~nm}$, this is from both Trp-214 and tyrosyl residues (Tyrs). The comparison between fluorescence quenching curves of glycated, defatted ((af)gHSA), and

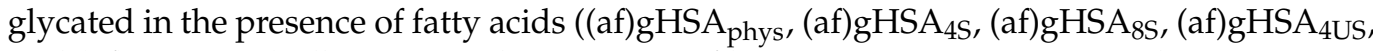
and (af)gHSA ${ }_{8 \mathrm{US}}$ ) albumins in the presence of losartan at $\lambda_{\mathrm{ex}}=275 \mathrm{~nm}$ and $\lambda_{\mathrm{ex}}=295 \mathrm{~nm}$ indicated the fluorophores involved in the interaction with the drug.

An almost identical course of albumin fluorescence quenching curves in LOS-(af)gHSA 4 US (Figure $3 \mathrm{e}$, in the insert) and LOS-(af)gHSA 8 (Figure $3 \mathrm{f}$, in the main view) system at $\lambda_{\mathrm{ex}}=275 \mathrm{~nm}$ and $\lambda_{\mathrm{ex}}=295 \mathrm{~nm}$ (almost a $4 \%$ difference in quenching of the intrinsic albumin fluorescence, Table 1) indicates the contribution of Trp-214 or its environment and a negligible contribution of Tyrs in the interaction of LOS with both, (af)gHSA 4 Us and (af) HSSA $_{8 \mathrm{~S}}$ in the environment of binding site. The human serum albumin contains only one tryptophanyl group.

It can be argued that LOS interacts with albumin containing four-times higher amounts of unsaturated ((af)gHSA 4 US $)$ and eight-times higher amounts of saturated ((af)gHSA 8 S) fatty acids in relation to the physiological concentration mainly in subdomain IIA, but the possibility of LOS interaction with albumin other sites cannot be excluded.

Differences in the course of quenching fluorescence at both excitation wavelengths $\lambda_{\mathrm{ex}}=275 \mathrm{~nm}$ and $\lambda_{\mathrm{ex}}=295 \mathrm{~nm}$ (almost 8\% in LOS-(af)gHSA (Figure 3d, in the main view) and LOS-(af)gHSA 8 US (Figure $3 \mathrm{f}$, in the insert), $12 \%$ in LOS-(af)gHSA 4 (Figure $3 \mathrm{e}$, in the main view), and more than $18 \%$ in LOS-(af)gHSA phys (Figure $3 \mathrm{~d}$, in the insert) system (Table 1)), indicate the simultaneous participation of the Trp-214 residue located in subdomain IIA and Tyrs residues located in the IIA, IB, and IIB and subdomains in the interaction of LOS with albumin at the appropriate binding site. As reported in the literature, tyrosyl residues in position 401 (Tyr-401) and 411 (Tyr-411) located in the IIIA subdomain of albumin play a major role in drug binding $[19,26]$. The fluorescence quenching technique is not sufficient to indicate which Tyrs moieties are involved in LOS binding.

The mechanism of losartan interaction with albumin can be determined on the basis of Stern-Volmer curves (Equation (2)). Based on the data obtained from glycated, defatted (af)gHSA and glycated in the presence of fatty acids (af)gHSA phys, (af)gHSA 4 , (af)gHSA 8 , (af)gHSA 4 US, and (af)gHSA 8 US albumin in the presence of LOS, the Stern-Volmer curves were plotted, $\lambda_{\mathrm{ex}}=275 \mathrm{~nm}$ (Figure $4 \mathrm{a}-\mathrm{c}$ ) and $\lambda_{\mathrm{ex}}=295 \mathrm{~nm}$ (Figure $4 \mathrm{~d}-\mathrm{f}$ ). The dashed lines indicate a model rectilinear course of Stern-Volmer dependence $\left(\mathrm{F}_{0} / \mathrm{F}=f\left(\left[\mathrm{C}_{\mathrm{LOS}}\right]\right)\right.$.

The Stern-Volmer curves obtained for the LOS-(af)gHSA, LOS-(af)gHSA 4 , and LOS(af)gHSA 8 system show a different course of curves plotted for the LOS-(af)gHSA phys (Figure $4 \mathrm{a}, \mathrm{d}$ ), LOS-(af)gHSA 4 US (Figure $4 \mathrm{~b}, \mathrm{e}$ ), and LOS-(af)gHSA 8 Us (Figure 4c,f) systems at both excitations $\lambda_{\mathrm{ex}}=275 \mathrm{~nm}$ and $\lambda_{\mathrm{ex}}=295 \mathrm{~nm}$. Higher fluorescence quenching $\left(\mathrm{F}_{0} / \mathrm{F}\right)$ for the whole range of losartan concentrations occurred for glycated albumin (af)gHSA $\mathrm{phys}_{\mathrm{s}}$ in the presence of physiologically fatty acids and albumin containing four- and eight-times higher amounts of saturated fatty acids ((af)gHSA 45 and (af)gHSA 8 ) in relation to the physiological concentration compared to the $\mathrm{F}_{0} / \mathrm{F}$ values obtained for glycated, defatted (af)gHSA albumin and containing four- and eight-times higher amounts of unsaturated fatty acids ((af)gHSA 4 US, (af)gHSA $8 \mathrm{US}$ ) in relation to the physiological concentration (Figure 4). 


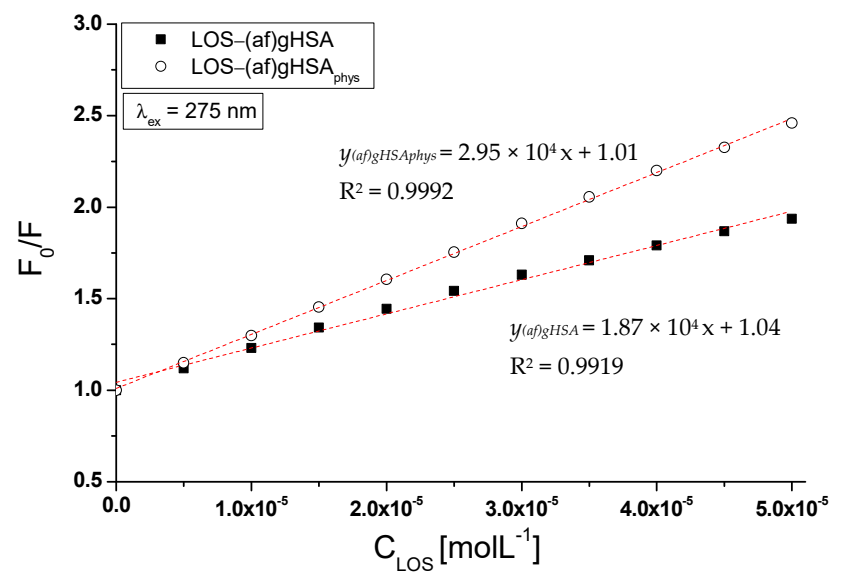

(a)

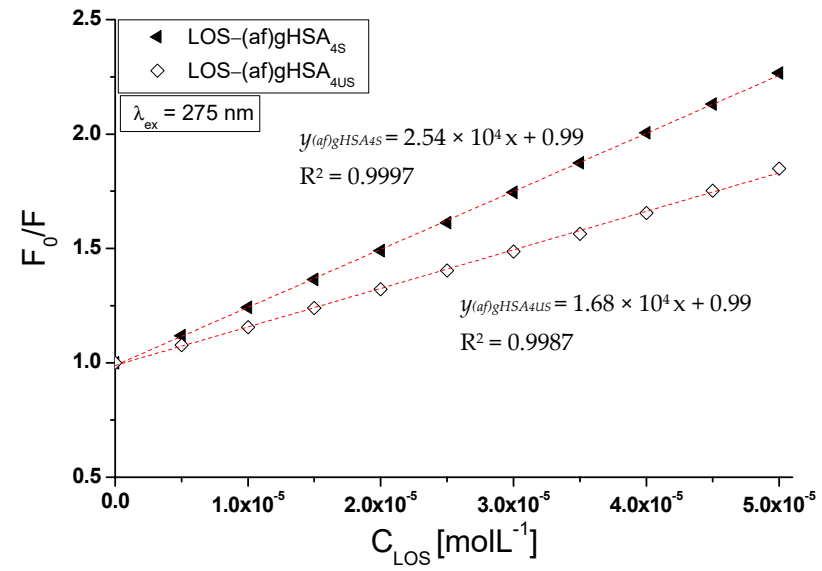

(b)

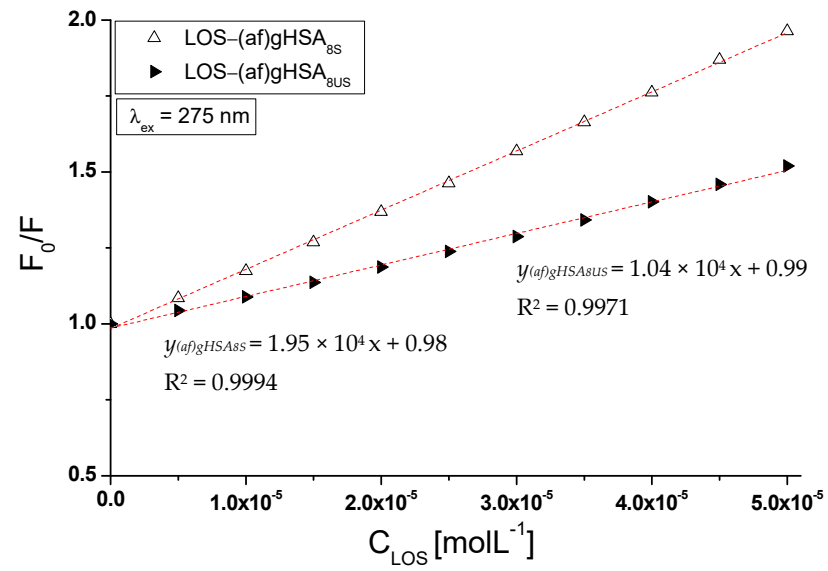

(c)

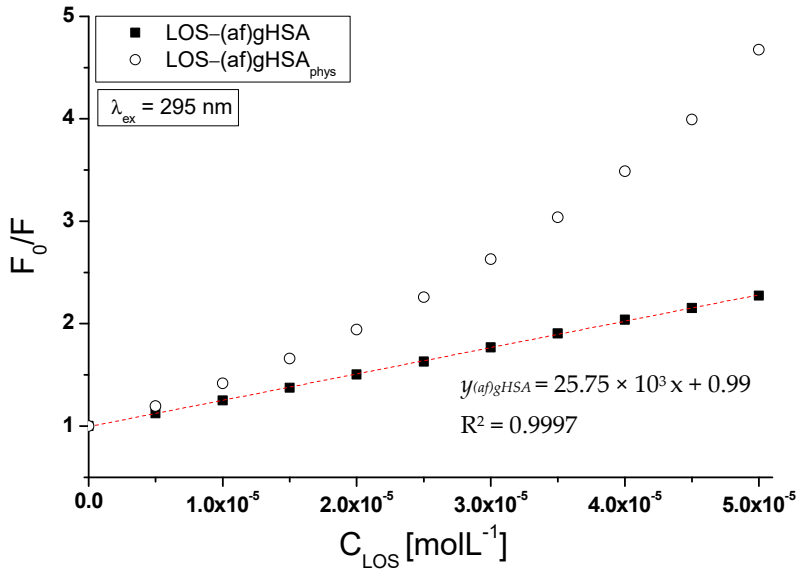

(d)

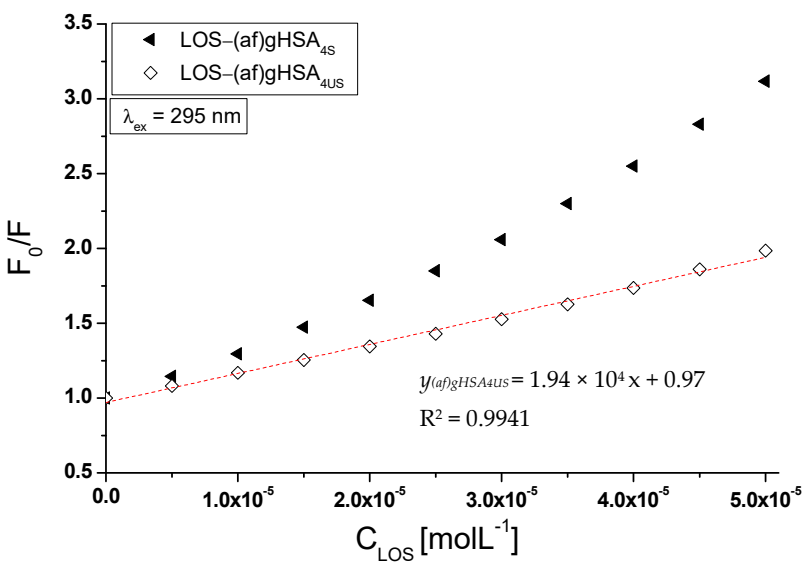

(e)

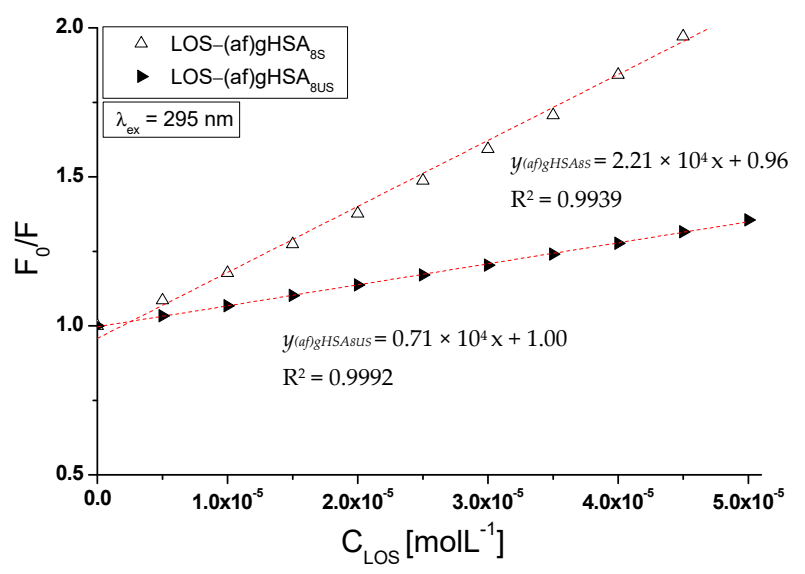

(f)

Figure 4. The Stern-Volmer curves for (a,d) LOS-(af)gHSA and LOS-(af)gHSA phys, (b,e) LOS(af)gHSA 4 S and LOS-(af)gHSA 4 US, (c,f) LOS-(af)gHSA 8 and LOS-(af)gHSA $8 \mathrm{US} ;(\mathbf{a}-\mathbf{c}) \lambda_{\mathrm{ex}}=275 \mathrm{~nm}$, (d-f) $\lambda_{\mathrm{ex}}=295 \mathrm{~nm}$; the error bars are smaller than the symbols.

Positive deviation (deviation in the OY direction) from the rectilinear relationship

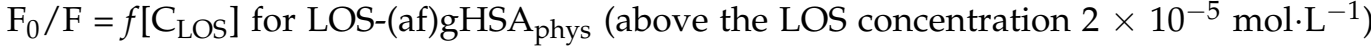
(Figure 4d) and LOS-(af)gHSA 4 (above the LOS concentration $2.5 \times 10^{-5} \mathrm{~mol} \cdot \mathrm{L}^{-1}$ ) (Figure 4e) system at $\lambda_{\mathrm{ex}}=295 \mathrm{~nm}$, indicates the occurrence of both dynamic and static quenching fluorescence of (af)gHSA $\mathrm{ghys}_{\text {and }}$ (af)gHSA $4 \mathrm{~S}$ albumin by losartan. During dynamic quenching, the ligand penetrates the environment of the macromolecule, and fluo- 
rescence quenching is caused by the collision of the quencher molecule and the fluorophore (Trp-214) of albumin in excitation state.

On the other hand, static quenching leads to a reduction in fluorescence intensity when the ligand binds to the fluorophore molecule in its basic state (unexcited), reducing the population of excitable fluorophores [20]. The existence of dynamic and static quenching of human serum albumin fluorescence was obtained in our previous studies when the influence of piracetam (as a potential glycation inhibitor) on gliclazide-glycated albumin interaction was analyzed [27].

The linear $\mathrm{F}_{0} / \mathrm{F}=f\left[\mathrm{C}_{\mathrm{LOS}}\right]$ relationship for the other systems (Figure 4), indicates a dynamic or static mechanism of macromolecule fluorescence quenching in the environment of subdomains containing amino acid residues that are involved in the formation of the ligand-albumin complex. Moreover, the order of fluorescence quenching rate constants $\mathrm{k}_{\mathrm{q}}$ equals to $10^{12}$ determined for LOS-glycated albumin system clearly indicates a static fluorescence quenching mechanism (Table 2), while according to Lakowicz, when the maximum value of the $\mathrm{k}_{\mathrm{q}}$ constant in the aqueous solution equals to $1 \times 10^{10}\left(\mathrm{~mol}^{-1} \cdot \mathrm{L} \cdot \mathrm{s}^{-1}\right)$, the dynamic fluorescence quenching mechanism occurs [19].

Table 2. Stern-Volmer constants $\mathrm{K}_{\mathrm{SV}}\left(\mathrm{mol}^{-1} \cdot \mathrm{L}\right)$, bimolecular quenching rate constants $\mathrm{k}_{\mathrm{q}}$ $\left(\mathrm{mol}^{-1} \cdot \mathrm{L} \cdot \mathrm{s}^{-1}\right)$ and maximum available fluorescence fraction $\mathrm{f}_{\mathrm{a}}$ of all albumin fluorophores calculated for the LOS-(af)gHSA, LOS-(af)gHSA ${ }_{\text {phys, }}$ LOS-(af)gHSA ${ }_{4 S}$, LOS-(af)gHSA 4 US, LOS-(af)gHSA 8 , and LOS-(af)gHSA ${ }_{8 \mathrm{US}}$ systems; $\lambda_{\mathrm{ex}}=275 \mathrm{~nm}$ and $\lambda_{\mathrm{ex}}=295 \mathrm{~nm}$.

\begin{tabular}{|c|c|c|c|}
\hline$\lambda_{\mathrm{ex}}=275 \mathrm{~nm}$ & $\mathrm{~K}_{\mathrm{SV}} \underset{\left(\mathrm{mol}^{-1} \cdot \mathrm{L}\right)}{ \pm \mathrm{RSD}^{*)}} \times 10^{4}$ & $\begin{array}{c}{ }^{\mathrm{a}} \mathrm{k}_{\mathrm{q}} \pm \mathrm{RSD}{ }^{*)} \times 10^{12} \\
\left(\mathrm{~mol}^{-1} \cdot \mathrm{L} \cdot \mathrm{s}^{-1}\right)\end{array}$ & $\mathbf{f}_{\mathrm{a}} \pm \mathrm{RSD}^{*)}$ \\
\hline LOS-(af)gHSA & $1.87 \pm 0.05$ & $3.12 \pm 0.08$ & $0.96 \pm 0.02$ \\
\hline LOS-(af)gHSA phys & $2.95 \pm 0.03$ & $4.92 \pm 0.06$ & $0.99 \pm 0.01$ \\
\hline LOS-(af)gHSA & $2.54 \pm 0.01$ & $4.23 \pm 0.01$ & $1.01 \pm 0.01$ \\
\hline LOS-(af)gHSA 85 & $1.95 \pm 0.02$ & $3.25 \pm 0.03$ & $1.02 \pm 0.01$ \\
\hline LOS-(af)gHSA 4 US & $1.68 \pm 0.02$ & $2.80 \pm 0.03$ & $1.01 \pm 0.01$ \\
\hline LOS-(af)gHSA 8 US & $1.04 \pm 0.02$ & $1.73 \pm 0.03$ & $1.01 \pm 0.01$ \\
\hline$\lambda_{\mathrm{ex}}=295 \mathrm{~nm}$ & $\mathrm{~K}_{\mathrm{SV}} \underset{\left(\mathrm{mol}^{-1} \cdot \mathrm{L}\right)}{\left. \pm \mathrm{RSD}^{*}\right)} \times 10^{4}$ & $\begin{array}{c}{ }^{\mathrm{a}} \mathrm{k}_{\mathrm{q}} \pm \mathrm{RSD}^{*)} \times 10^{12} \\
\left(\mathrm{~mol}^{-1} \cdot \mathrm{L} \cdot \mathrm{s}^{-1}\right)\end{array}$ & $\mathbf{f}_{\mathbf{a}} \pm \mathbf{R S D}^{*)}$ \\
\hline LOS-(af)gHSA & $2.57 \pm 0.02$ & $4.28 \pm 0.03$ & $1.01 \pm 0.01$ \\
\hline LOS-(af)gHSA phys & $2.65 \pm 0.01$ & $4.42 \pm 0.01$ & $1.39 \pm 0.01$ \\
\hline LOS-(af)gHSA 45 & $2.08 \pm 0.02$ & $3.47 \pm 0.03$ & $1.34 \pm 0.02$ \\
\hline LOS-(af)gHSA 85 & $2.22 \pm 0.06$ & $3.70 \pm 0.09$ & $1.04 \pm 0.01$ \\
\hline LOS-(af)gHSA 4 US & $1.94 \pm 0.05$ & $3.23 \pm 0.08$ & $1.03 \pm 0.01$ \\
\hline LOS-(af)gHSA 8 US & $0.71 \pm 0.01$ & $1.18 \pm 0.01$ & $1.00 \pm 0.01$ \\
\hline
\end{tabular}

*) relative standard deviation; ${ }^{a}$ calculated using: $\mathrm{k}_{\mathrm{q}}=\frac{\mathrm{K}_{\mathrm{SV}}}{\tau_{0}}$, where $\tau_{0}=6.0 \times 10^{-9} \mathrm{~s}[22]$-the average fluorescence lifetime of albumin without quencher.

From the $\mathrm{F}_{0} / \mathrm{F}=f\left[\mathrm{C}_{\mathrm{LOS}}\right]$ relationship for a system with a linear course of the SternVolmer curve, the Stern-Volmer constants $\mathrm{K}_{\mathrm{SV}}$, the bimolecular quenching rate constants $\mathrm{k}_{\mathrm{q}}$ and maximum available fluorescence fraction $\mathrm{f}_{\mathrm{a}}$ of all albumin fluorophores were determined. Quenching parameters $\left(\mathrm{K}_{\mathrm{SV}}, \mathrm{k}_{\mathrm{q}}=\mathrm{K}_{\mathrm{SV}} / \tau_{0}\right)$ and $\mathrm{f}_{\mathrm{a}}$ for a system with non-linear Stern-Volmer relationship (LOS-(af)gHSA phys (Figure 4d) and LOS-(af)gHSA 4 (Figure 4e) for $\left.\lambda_{\mathrm{ex}}=295 \mathrm{~nm}\right)$ were determined from $\mathrm{F}_{0} / \Delta \mathrm{F}=f\left(1 /\left[\mathrm{C}_{\mathrm{LOS}}\right]\right)$ relationship represented by Stern-Volmer equation modified by Lehrer (Equation (3)) [21]. The plot of $\mathrm{F}_{0} / \Delta \mathrm{F}$ vs. $1 /\left[C_{L O S}\right]$ is found to be linear with the intercept on the ordinate (Figure $5 a, b$ ). The reciprocal of the intercept gives the value of $f_{a}$ while the intercept/slope gives the value of the Stern-Volmer constants KSV . The obtained results have been collected in Table 2. 


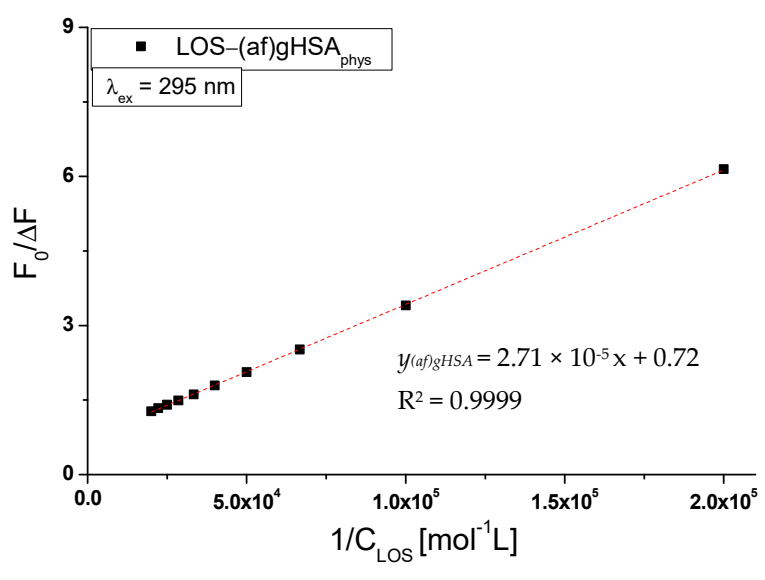

(a)

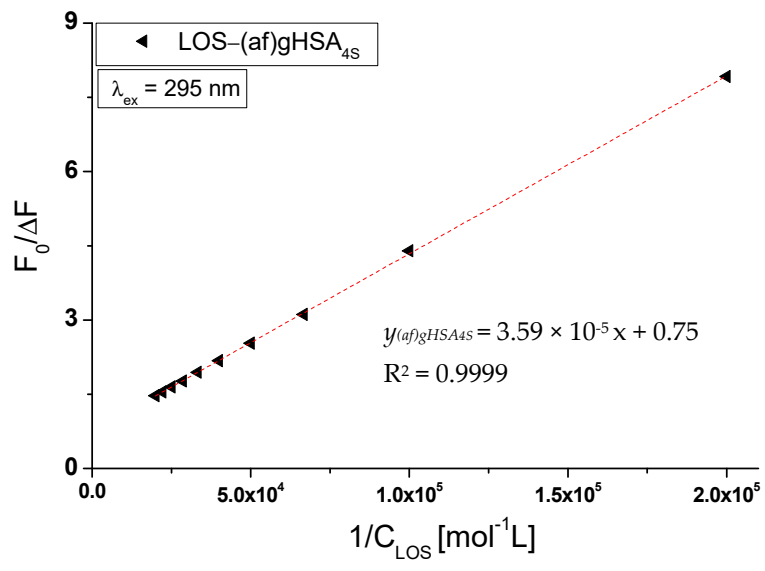

(b)

Figure 5. The Stern-Volmer curves modified by Lehrer for (a) LOS-(af)gHSA phys and (b) LOS(af)gHSA 4 system; $\lambda_{\text {ex }}=295 \mathrm{~nm}$; the error bars are smaller than the symbols.

The Stern-Volmer constant is used to assess the availability of the quencher to the excited fluorophore. The growth of $\mathrm{K}_{\mathrm{SV}}$ value is associated with the increase of ligand molecule availability to the macromolecule and the formation of the complex in an excited state. As can be seen in the Table 2, the higher values of $\mathrm{K}_{\mathrm{SV}}$ constant obtained for the LOS-

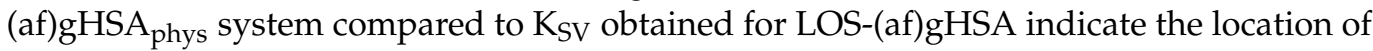
losartan molecules closer to the fluorophores of glycated, fatted by fatty acids physiological mixture albumin ((af)gHSA phys) than glycated, defatted albumin (af)gHSA fluorophores.

The presence of fatty acids physiological mixture in glycated human serum albumin probably makes formation of LOS-(af)gHSA ${ }_{\text {phys }}$ complex easier than the absence of fatty acids in the system (especially when the observed emission of fluorescence comes from both Trp-214 and tyrosyl residues (Tyrs)). The Stern-Volmer values and biomolecular quenching rate constants obtained for LOS-(af)gHSA 4 and LOS-(af)gHSA 8 are higher than $\mathrm{K}_{\mathrm{SV}}$ and $\mathrm{k}_{\mathrm{q}}$ values obtained for LOS-(af)gHSA 4 US and LOS-(af)gHSA 8 US $\left(\lambda_{\mathrm{ex}}=275 \mathrm{~nm}\right.$ and $\lambda_{\mathrm{ex}}=295 \mathrm{~nm}$ ).

Moreover, a two-fold increase in the amount of saturated fatty acids in the LOSalbumin system resulted in $23 \%$ decrease of $\mathrm{K}_{\mathrm{SV}}$ constant for $\lambda_{\mathrm{ex}}=275 \mathrm{~nm}$ and only $6 \%$ increase Ksv for $\lambda_{\mathrm{ex}}=295 \mathrm{~nm}$. On the other hand, a two-fold increase in the amount of unsaturated fatty acids in the LOS-albumin system caused $38 \%$ and $63 \%$ decreases in $\mathrm{K}_{\mathrm{SV}}$ for $\lambda_{\mathrm{ex}}=275 \mathrm{~nm}$ and $\lambda_{\mathrm{ex}}=295 \mathrm{~nm}$, respectively. These results indicate that LOS molecules locate at different distances to fluorophores of glycated albumin containing various amounts of saturated and unsaturated fatty acids. In addition, it can be seen that the availability of albumin (af)gHSA $\mathrm{ghys}_{\text {and }}$ (af)gHSA 4 fluorophores (especially Trp-214 residues) for individual LOS binding sites is significantly facilitated (Table 2).

To determine the nature of the interaction of losartan with glycated, defatted (af)gHSA and glycated in the presence of fatty acids albumin (af)gHSA ${ }_{\text {phys }},(\mathrm{af}) \mathrm{gHSA}_{4 \mathrm{~S}}$, (af)gHSA $8 \mathrm{~S}$, (af)gHSA 4 US, and (af)gHSA 8 sus, binding isotherms were plotted in the LOS-(af)gHSA and

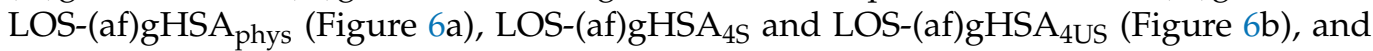
LOS-(af)gHSA $8 \mathrm{~S}$ and LOS-(af)gHSA $8 \mathrm{US}$ (Figure 6c) systems, $\lambda_{\mathrm{ex}}=275 \mathrm{~nm}$ (Figure 6, in the main view) and $\lambda_{\mathrm{ex}}=295 \mathrm{~nm}$ (Figure 6, in the insert).

Similarly, as in our previous paper [28], where the interaction of tolbutamide and losartan with human serum albumin in hyperglycemia states were studied, a non-linear relationship $\mathrm{r}=f\left(\left[\mathrm{~L}_{\mathrm{f}}\right]\right)$ was observed (Figure 6). The non-linear shape of the binding isotherms obtained for LOS-(af)gHSA and LOS-(af)gHSA ${ }_{\text {phys }}$ (Figure 6a), LOS-(af)gHSA 45 and LOS-(af)gHSA 4 US (Figure 6b), and LOS-(af)gHSA 8 (Figure 6c) complexes indicates the mixed (specific and non-specific) nature of losartan interaction with macromolecules. 


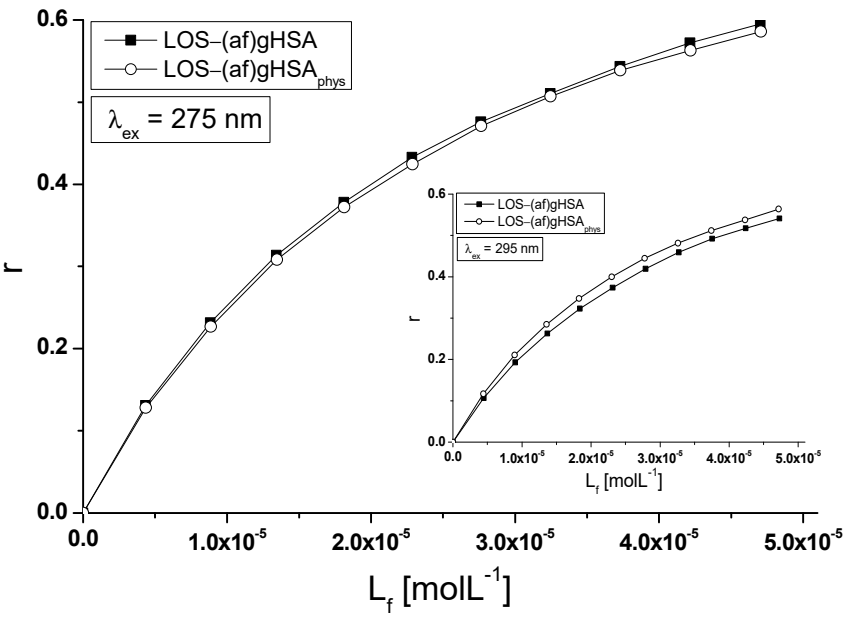

(a)

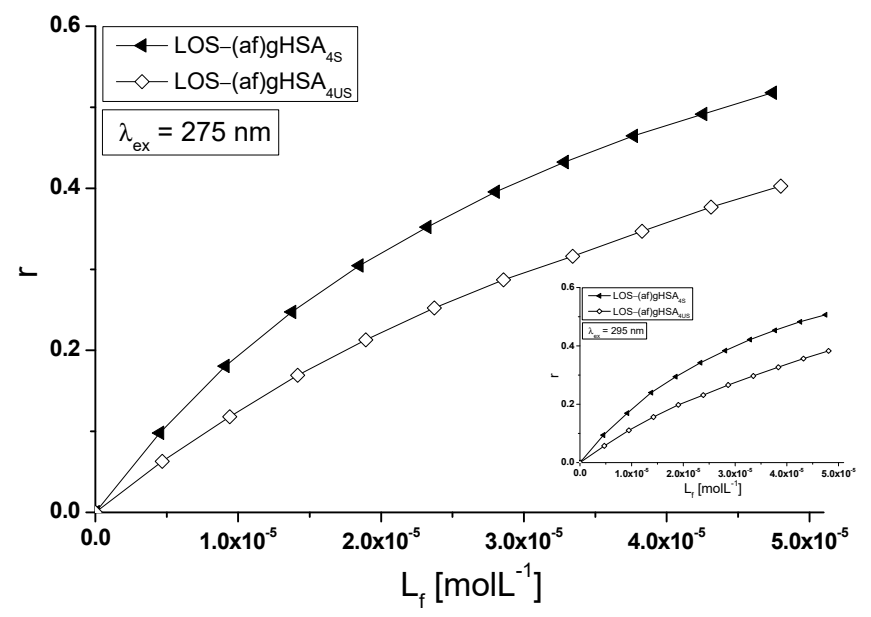

(b)

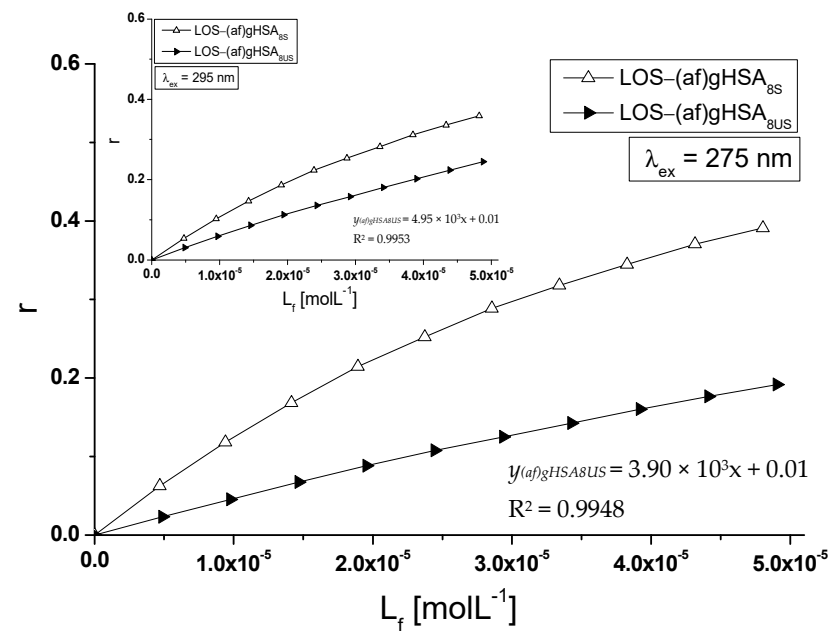

(c)

Figure 6. Binding isotherms of (a) (af)gHSA, (af)gHSA $\mathrm{ghys}$, (b) (af)gHSA 4 , (af)gHSA 4 US, and (c) ${\text { (af) } \mathrm{gHSA}_{8 \mathrm{~S}},(\mathrm{af}) \mathrm{gHSA}}_{8 \mathrm{US}}$ at $5 \times 10^{-6} \mathrm{~mol} \cdot \mathrm{L}^{-1}$ concentration with LOS at $5 \times 10^{-6}-5 \times 10^{-5}$ $\mathrm{mol} \cdot \mathrm{L}^{-1}$ concentration, $\lambda_{\mathrm{ex}}=275 \mathrm{~nm}$ (in the main view), $\lambda_{\mathrm{ex}}=295 \mathrm{~nm}$ (in the insert); the error bars are smaller than the symbols.

This means that losartan binds not only to its specific binding sites in glycated, defatted and in the presence of fatty acids albumin but also non-specifically interacts with the hydrophobic fragments of its surface [29]. However, the shape of the binding isotherms for glycated human serum albumin with fatty acids containing eight-times more unsaturated fatty acids in relation to the physiological value, indicates the occurrence of only the non-specific nature of losartan binding to (af)gHSA 8 Us (Figure 6c).

Specific binding is characterized by high affinity and low binding capacity, while nonspecific binding is characterized by low affinity and unlimited drug binding capacity [29]. Regardless of the course of binding isotherms (Figure 6), the losartan-glycated albumin interaction is likely characterized by a specific type of binding because, in physiological environments, the drug:albumin molar ratio is much smaller than 1:1 and equals to 1:500.

There are many methods for the calculation of association constant $\left(K_{a}\right)$ that characterizes the stability of formed drug-albumin complex for the determination the number of drug molecules $(n)$ associated with one albumin molecule at equilibrium, or for the prediction an existence of one or more independent classes of binding sites. In the present work, specific binding of losartan to glycated human serum albumin in LOS-(af)gHSA, LOS(af)gHSA phys $_{\text {, LOS-(af)gHSA }}$, LOS-(af)gHSA 4 Us, LOS-(af)gHSA 8 , and LOS-(af)gHSA 8 Us 
complexes has been quantitatively characterized using the association constant $\mathrm{K}_{\mathrm{a}}$ calculated based on the Scatchard (the dependence of $r /\left[L_{f}\right]$ on $r$, Equation (4), Figure 7) and the Klotz (the dependence of $1 / \mathrm{r}$ on $1 /\left[\mathrm{L}_{\mathrm{f}}\right]$, Equation (5), Figure 8) equations.

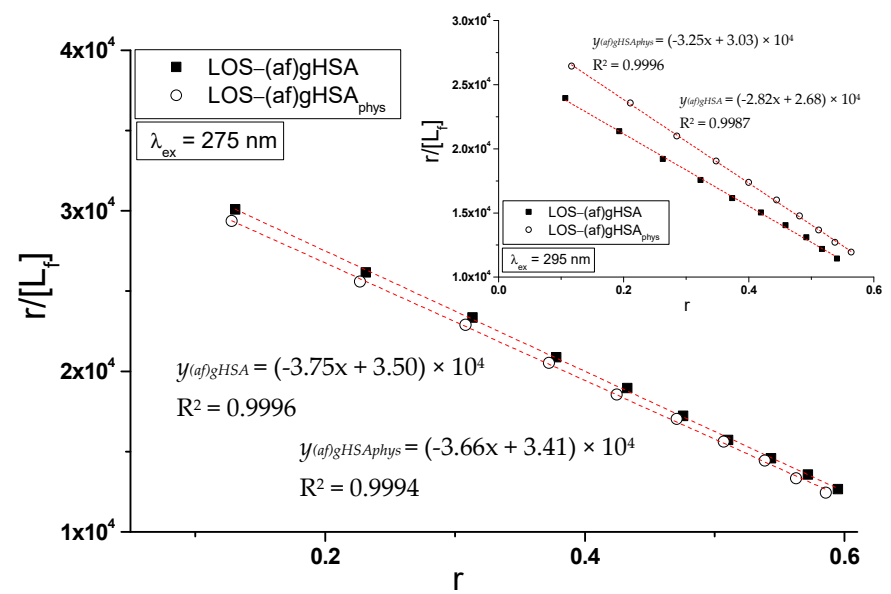

(a)

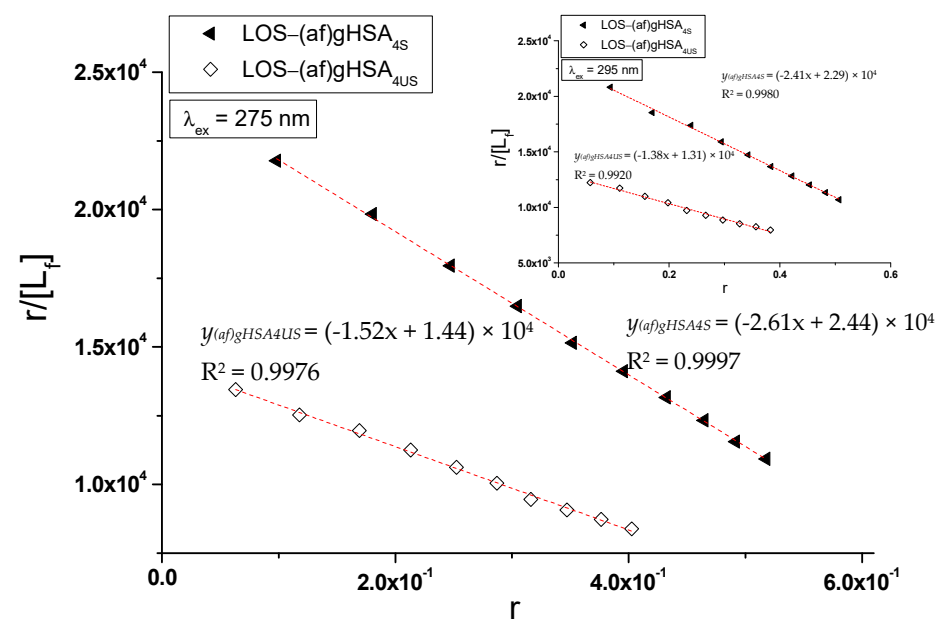

(b)

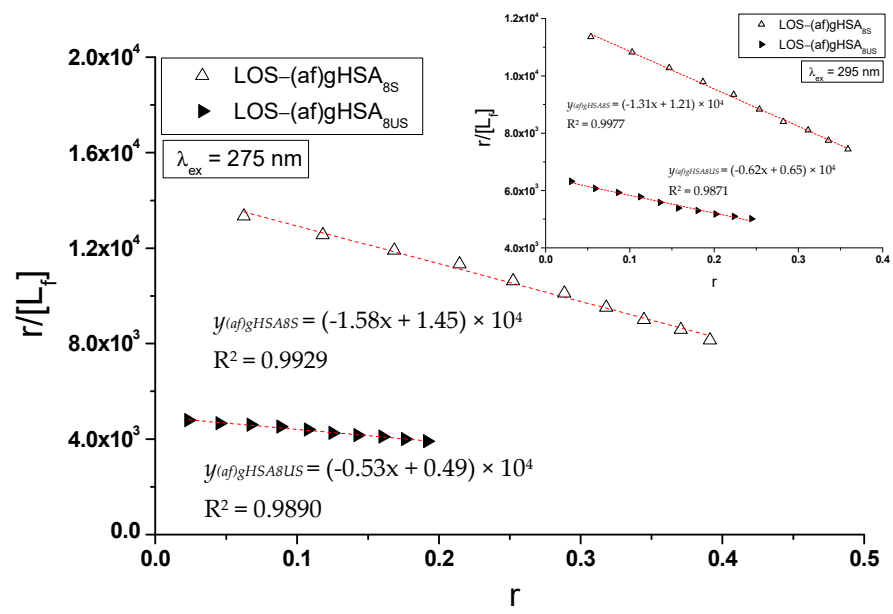

(c)

Figure 7. The Scatchard curves of $r /\left[\mathrm{L}_{\mathrm{f}}\right]$ vs. $\mathrm{r}$ for (a) LOS-(af)gHSA and LOS-(af)gHSA $\mathrm{A}_{\text {phys }}$, (b) LOS-(af)gHSA 4 and LOS-(af)gHSA 4 US, and (c) LOS-(af)gHSA 8 S and LOS-(af)gHSA 8 US systems, $\lambda_{\mathrm{ex}}=275 \mathrm{~nm}$ (in the main view), $\lambda_{\mathrm{ex}}=295 \mathrm{~nm}$ (in the insert); the error bars are smaller than the symbols. 


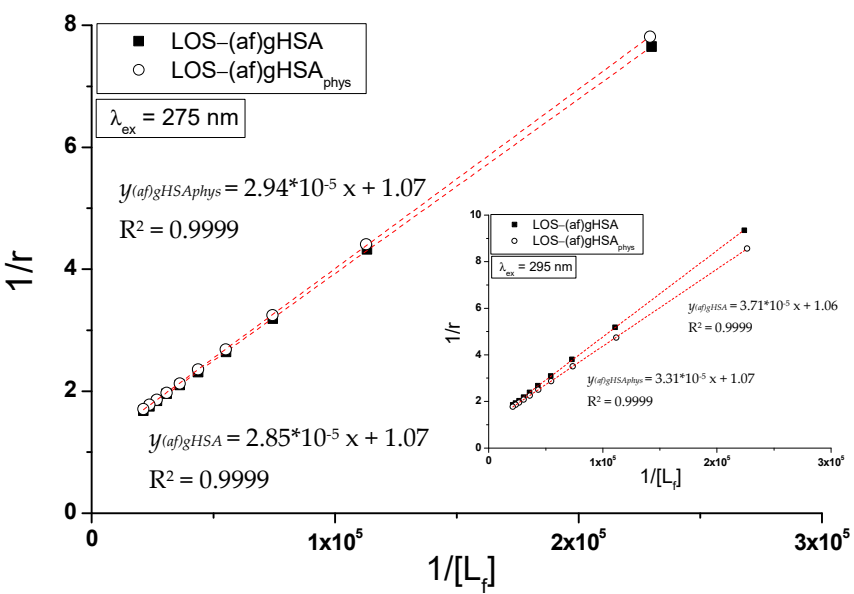

(a)

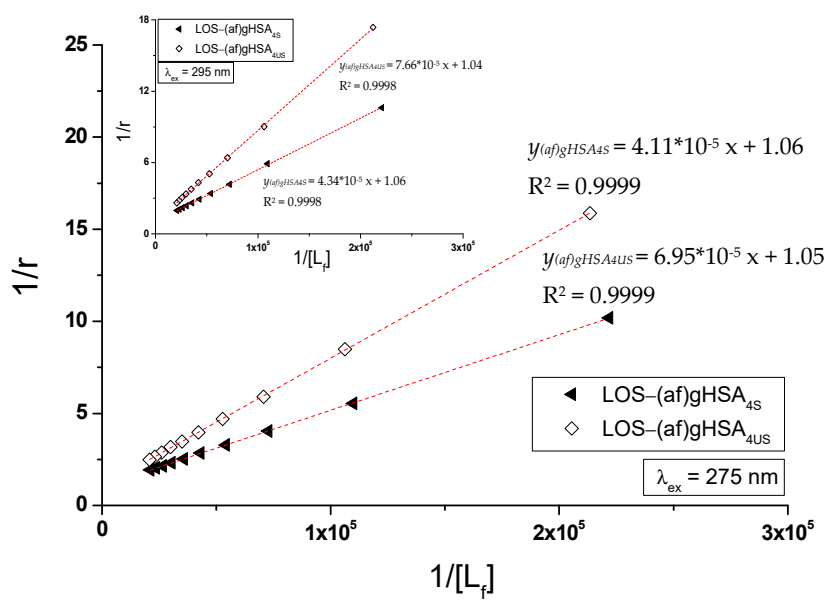

(b)

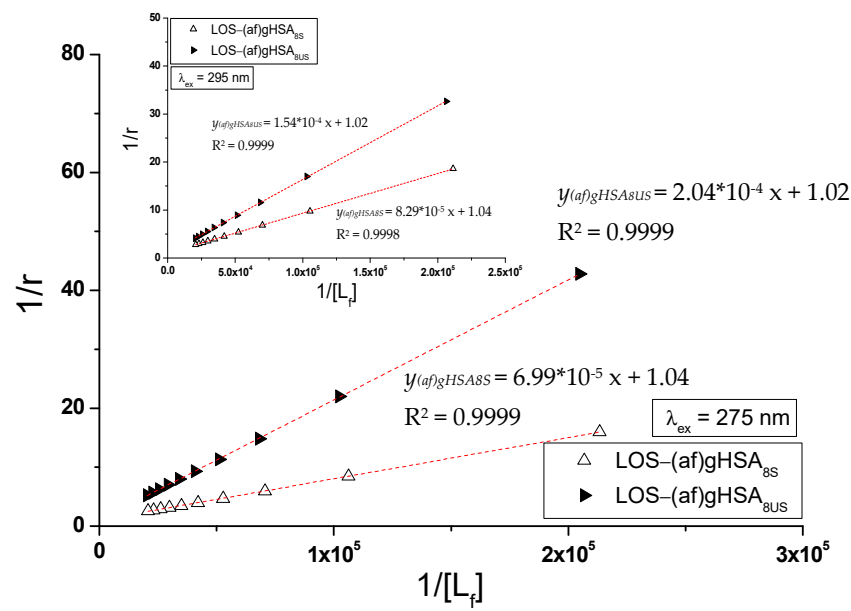

(c)

Figure 8. The Klotz curves of $1 / \mathrm{r}$ vs. $1 /\left[\mathrm{L}_{\mathrm{f}}\right]$ for (a) LOS-(af)gHSA and LOS-(af)gHSA ${ }_{\text {phys }}$ (b) LOS(af)gHSA 4 and LOS-(af)gHSA 4 US, and (c) LOS-(af)gHSA 8 and LOS-(af)gHSA 8 US systems, $\lambda_{\mathrm{ex}}=275 \mathrm{~nm}$ (in the main view), $\lambda_{\mathrm{ex}}=295 \mathrm{~nm}$ (in the insert); the error bars are smaller than the symbols.

In the Scatchard equation, the concentration of the bound ligand to the protein is the independent variable, while, in the Klotz equation, the independent variable is the reciprocal of the free ligand fraction. To study the possible cooperation of losartan binding to the macromolecule, the Hill interaction factors $\left(n_{H}\right)$ were determined by the use of the Hill equation (the dependence of $\log [r /(1-r)]$ on $\log \left[L_{f}\right]$, Equation (6), Figure 9). 
The number of losartan molecules $(n)$ forming a complex with one molecule of (af)gHSA, (af)gHSA ${ }_{\text {phys }}$, (af)gHSA 4 , (af)gHSA 4 US, (af)gHSA 8 , and (af)gHSA 8 Us at equilibrium state for a specific class of binding sites was also obtained. The binding parameters $\left(\mathrm{K}_{\mathrm{a}}, n\right)$ and Hill $\mathrm{n}_{\mathrm{H}}$ coefficient (interaction factor) are summarized in Table 3.

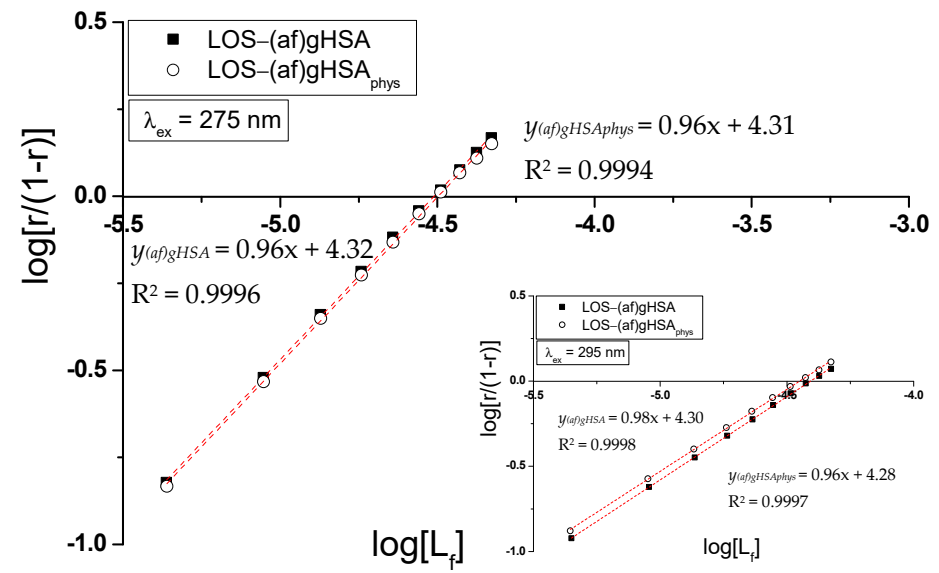

(a)

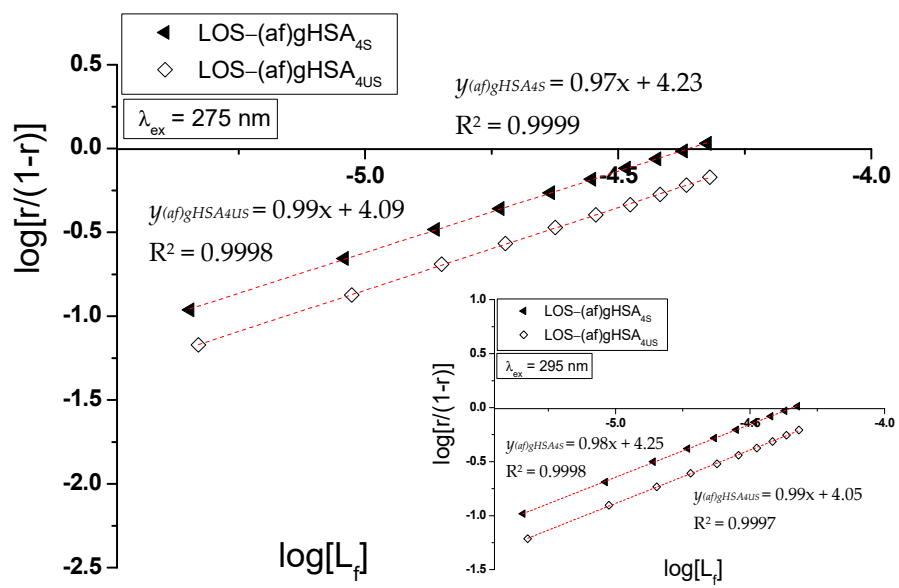

(b)

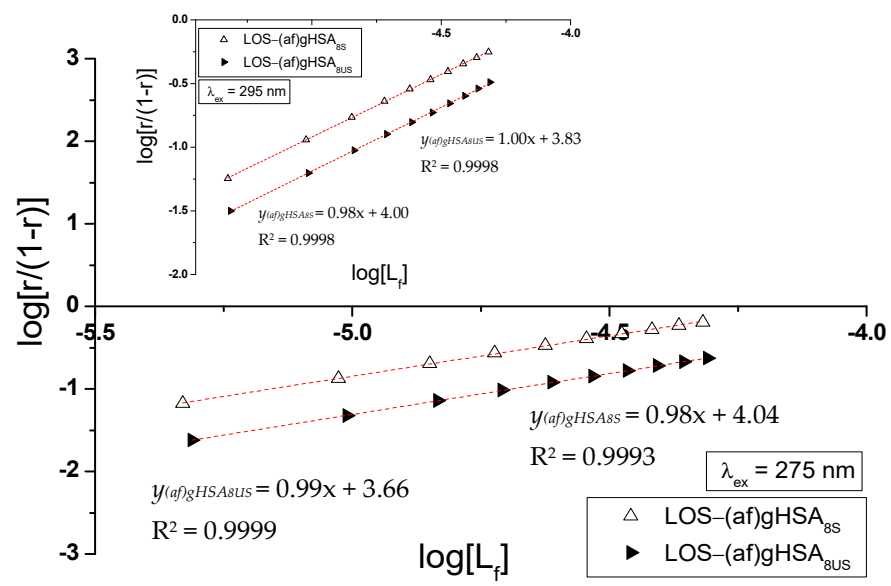

(c)

Figure 9. The Hill curves of $\log [\mathrm{r} /(1-\mathrm{r})]$ vs. $\log \left[\mathrm{L}_{\mathrm{f}}\right]$ for (a) LOS-(af)gHSA and LOS-(af)gHSA $\mathrm{phys}$ (b) LOS-(af)gHSA 4 S and LOS-(af)gHSA 4 US, and (c) LOS-(af)gHSA 8 s and LOS-(af)gHSA 8 US systems, $\lambda_{\mathrm{ex}}=275 \mathrm{~nm}$ (in the main view), $\lambda_{\mathrm{ex}}=295 \mathrm{~nm}$ (in the insert); the error bars are smaller than the symbols. 
Table 3. Association constants $\mathrm{K}_{\mathrm{a}}\left(\mathrm{mol}^{-1} \cdot \mathrm{L}\right)$, mean number of LOS moles bound with one mole of (af)gHSA, (af)gHSA phys (af)gHSA 4 , (af)gHSA 4 US, (af)gHSA 8 , and (af)gHSA 8 Us $(n)$, the Hill coefficient $\left(\mathrm{n}_{\mathrm{H}}\right)$ in The LOS-albumin systems; $\lambda_{\mathrm{ex}}=275 \mathrm{~nm}, \lambda_{\mathrm{ex}}=295 \mathrm{~nm}$.

\begin{tabular}{|c|c|c|c|c|c|}
\hline \multirow[b]{2}{*}{$\lambda_{\mathrm{ex}}=275 \mathrm{~nm}$} & \multicolumn{2}{|c|}{ Scatchard Method } & \multicolumn{2}{|c|}{ Klotz Method } & \multirow{2}{*}{$\begin{array}{l}\text { Hill Method } \\
\left.\mathbf{n}_{\mathbf{H}} \pm \mathrm{RSD}^{*}\right)\end{array}$} \\
\hline & $\begin{array}{c}\mathrm{K}_{\mathrm{a}} \pm \mathrm{RSD}^{*)} \\
\times 10^{4}\left(\mathrm{~mol}^{-1} \cdot \mathrm{L}\right)\end{array}$ & $n \pm \mathbf{R S D} *)$ & $\begin{array}{c}\mathrm{K}_{\mathrm{a}} \pm \mathrm{RSD}^{*)} \\
\times 10^{4}\left(\mathrm{~mol}^{-1} \cdot \mathrm{L}\right)\end{array}$ & $n \pm \mathrm{RSD}^{*)}$ & \\
\hline LOS-(af)gHSA & $3.75 \pm 0.02$ & $0.93 \pm 0.01$ & $3.74 \pm 0.01$ & $0.94 \pm 0.01$ & $0.96 \pm 0.01$ \\
\hline LOS-(af)gHSA phys & $3.66 \pm 0.03$ & $0.93 \pm 0.01$ & $3.64 \pm 0.01$ & $0.93 \pm 0.01$ & $0.96 \pm 0.01$ \\
\hline LOS-(af)gHSA $_{4 S}$ & $2.61 \pm 0.02$ & $0.94 \pm 0.01$ & $2.59 \pm 0.01$ & $0.94 \pm 0.01$ & $0.97 \pm 0.01$ \\
\hline LOS-(af)gHSA 8 S & $1.52 \pm 0.03$ & $0.95 \pm 0.02$ & $1.51 \pm 0.02$ & $0.95 \pm 0.01$ & $0.99 \pm 0.01$ \\
\hline LOS-(af)gHSA 4 US & $1.58 \pm 0.04$ & $0.92 \pm 0.03$ & $1.49 \pm 0.02$ & $0.96 \pm 0.02$ & $0.98 \pm 0.01$ \\
\hline LOS-(af)gHSA 8 US & $0.53 \pm 0.02$ & $0.94 \pm 0.04$ & $0.50 \pm 0.02$ & $0.99 \pm 0.04$ & $0.99 \pm 0.01$ \\
\hline$\lambda_{\mathrm{ex}}=295 \mathrm{~nm}$ & $\begin{array}{c}\mathrm{K}_{\mathrm{a}} \pm \mathrm{RSD}^{*)} \\
\times 10^{4}\left(\mathrm{~mol}^{-1} \cdot \mathrm{L}\right)\end{array}$ & $n \pm \mathrm{RSD} *)$ & $\begin{array}{c}\mathrm{K}_{\mathrm{a}} \pm \mathrm{RSD}^{*)} \\
\times 10^{4}\left(\mathrm{~mol}^{-1} \cdot \mathrm{L}\right)\end{array}$ & $n \pm \mathrm{RSD} *)$ & $\mathbf{n}_{\mathbf{H}} \pm \mathbf{R S D}^{*}$ \\
\hline LOS-(af)gHSA & $2.82 \pm 0.03$ & $0.95 \pm 0.02$ & $2.87 \pm 0.01$ & $0.94 \pm 0.01$ & $0.98 \pm 0.01$ \\
\hline LOS-(af)gHSA phys & $3.25 \pm 0.02$ & $0.93 \pm 0.01$ & $3.22 \pm 0.01$ & $0.94 \pm 0.01$ & $0.96 \pm 0.01$ \\
\hline LOS-(af)gHSA 45 & $2.41 \pm 0.04$ & $0.95 \pm 0.02$ & $2.44 \pm 0.03$ & $0.94 \pm 0.02$ & $0.98 \pm 0.01$ \\
\hline LOS-(af)gHSA & $1.38 \pm 0.04$ & $0.95 \pm 0.04$ & $1.36 \pm 0.03$ & $0.96 \pm 0.03$ & $0.99 \pm 0.01$ \\
\hline LOS-(af)gHSA 4 US & $1.31 \pm 0.02$ & $0.93 \pm 0.02$ & $1.26 \pm 0.02$ & $0.96 \pm 0.01$ & $0.98 \pm 0.01$ \\
\hline LOS-(af)gHSA 8 US & $0.62 \pm 0.02$ & $1.04 \pm 0.05$ & $0.67 \pm 0.02$ & $0.98 \pm 0.03$ & $1.00 \pm 0.01$ \\
\hline
\end{tabular}

*) relative standard deviation.

The straight-line Scatchard (Figure 7), Klotz (Figure 8) and Hill (Figure 9) plots indicate the presence of one independent class of losartan binding sites in the albumin (af)gHSA, (af)gHSA phys, (af)gHSA 4 , (af)gHSA 8 , (af)gHSA 4 Us, and (af)gHSA 8 us structure (or one binding site). The course of binding isotherms, which determine the binding specificity of ligand to albumin, for LOS-(af)gHSA 8 US system is linear (Figure 6c). The straight-line relationship of $\mathrm{r}=f\left(\left[\mathrm{~L}_{\mathrm{f}}\right]\right)$ Taira and Terada [29] explained by the non-specific interaction of ligand with the hydrophobic fragments of macromolecule surfaces.

The association constants $\mathrm{K}_{\mathrm{a}}$ determined from the Scatchard and the Klotz relationships for the complexes LOS-(af)gHSA 8 US for $\lambda_{\mathrm{ex}}=275 \mathrm{~nm}$ and $\lambda_{\mathrm{ex}}=295 \mathrm{~nm}$ prove the specific nature of losartan binding within the albumin (Table 3). For LOS-(af)gHSA complex, the association constants $K_{a}$ are the same (for $\lambda_{\mathrm{ex}}=275 \mathrm{~nm}$ ) and not much lower (for $\lambda_{\text {ex }}=295 \mathrm{~nm}$ ) than the constants $K_{a}$ values obtained for losartan-(af)gHSA ${ }_{\text {phys }}$ complex (Table 3), which indicates that LOS has the same affinity for (af)gHSA and (af)gHSA ${ }_{\text {phys }}$ albumin binding sites.

For the LOS-albumin complex with a two-times greater amount of saturated ((af)gHSA 8 ) and unsaturated ((af)gHSA $8 \mathrm{US})$ fatty acids, the $\mathrm{K}_{\mathrm{a}}$ constants are smaller than those obtained for the LOS-(af)gHSA 4 S and LOS-(af)gHSA 4 US for $\lambda_{\mathrm{ex}}=275 \mathrm{~nm}$ and $295 \mathrm{~nm}$ (Table 3). This means that a higher concentration of both saturated and unsaturated fatty acids in glycated albumin reduces the stability of the complex formed with losartan. For LOS-(af)gHSA, LOS(af)gHSA phys $_{\text {, LOS-(af)gHSA }}$, LOS-(af)gHSA 8 , LOS-(af)gHSA 4 Us, and LOS-(af)gHSA 8 Us complexes, an average of one ligand molecule binds to one albumin molecule $(n \approx 1)$.

The Hill interaction coefficient $\mathrm{n}_{\mathrm{H}}$ equals unity $\left(\mathrm{n}_{\mathrm{H}} \approx 1\right)$ and indicates a lack of cooperativity in the binding of LOS to albumins in the vicinity of Trp-214 and Tyrs residues. This is the same value of $\mathrm{n}_{\mathrm{H}}$ that we obtained in our previous work [23] when we determined the Hill interaction coefficient for acetohexamide-albumin complex with four- and eightfold higher unsaturated and eight-fold higher saturated fatty acids amount compared to physiological value.

Based on the in vitro results, the fatty acids affect losartan binding to glycated human serum albumin. It can be assumed that, under conditions of abnormal fat content in the body, the pharmacokinetics of the drug may be disturbed. It is noteworthy that during a treatment with losartan it is important to control the amount of fatty acids supplied to the body with diet and/or in the form of supplements. Stronger binding of LOS to albumin weakens its therapeutic effect; however, on the other hand, the free drug fraction has potentially toxic side effects that can be dangerous to the patient's health. The research 
suggests the need for individual dose selection, especially for the obese patients with chronic diseases.

\subsection{Structural Modification of Glycated Human Serum Albumin Caused by Fatty Acids}

The physicochemical and biological properties of proteins are directly dependent on their spatial structure. It is for this reason that studies that allow us to observe conformational changes in protein caused by various modifications are important. A number of structural modifications, especially in the tertiary confirmation of human serum albumin, are attributed to, e.g., protein glycation [30].

In this part of the study, we examined whether fatty acids cause additional conformational changes in the tertiary structure of glycated albumin, which simulates diabetes in the body. It is of key importance in planning therapy because the strength and nature of the drug's interactions with its main distributor may change in the presence of coexisting diabetes and obesity (Section 2.1).

Although circular dichroism (CD) plays an important role in the study of protein folding as it allows the characterization of secondary and tertiary structure of proteins in native, unfolded and partially folded states [31], the CD spectra of the studied proteins were impossible to register due to the presence of the introduced fatty acids. Hence, to indicate changes in the tertiary structure of glycated albumin induced by the presence of fatty acids, fluorescence spectroscopy was used.

For this purpose, we compared the emission and synchronous fluorescence spectra of glycated human serum albumin ((af)gHSA) in the absence and in the presence of fatty acids (FA) corresponding to the physiological FA composition in human blood ((af)gHSA $\left.\mathrm{phys}_{\text {s }}\right)$ and in various clinical states, proceeding with increased concentrations of saturated ((af)gHSA $4 \mathrm{~S}$ and (af)gHSA 8 S) and unsaturated FA ((af)gHSA 4 US and (af)gHSA $\left.{ }_{8 \mathrm{US}}\right)$ compared to physiological values. Additionally, based on the Red Edge Excitation Shift (REES) analysis, the spatial organization of the Trp-214 molecules was determined.

Synchronous (Figures 10-12, main view) and emission (Figures 10-12, insert) fluorescence spectra of (af)gHSA and (af)gHSA phys (Figure 10); (af)gHSA, (af)gHSA 4 and (af)gHSA 4 Us (Figure 11); (af)gHSA, (af)gHSA 8 and (af)gHSA 8 Us (Figure 12) were used to show the conformational changes in the environment of the tryptophanyl and tyrosine residues of glycated human serum albumin influenced by fatty acids. It is well known that the wavelength of $275 \mathrm{~nm}$ excites not only Trp-214 but also tyrosine residues and it is impossible to separately observe the fluorescence of these fluorophores.

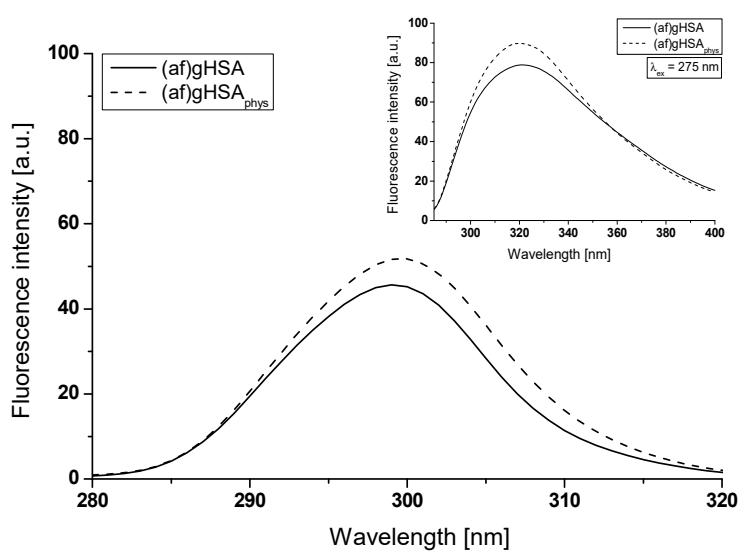

(a)

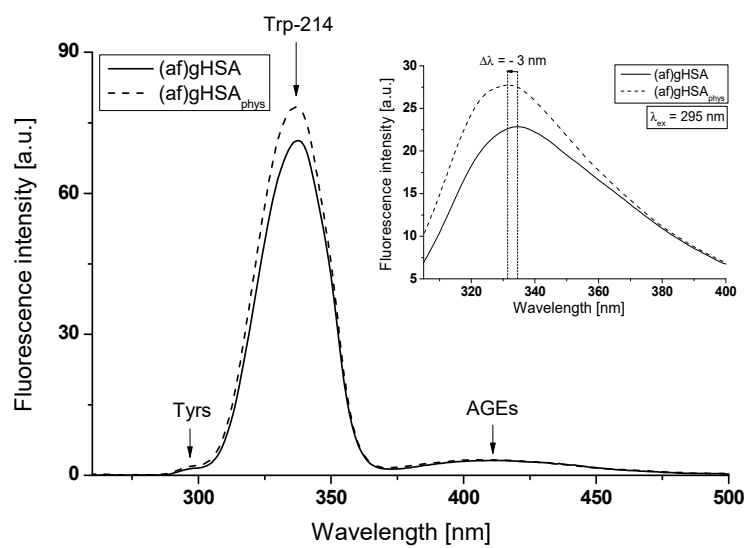

(b)

Figure 10. Main view: synchronous fluorescence spectra of (af)gHSA and (af)gHSA phys at $5 \times 10^{-6} \mathrm{~mol} \cdot \mathrm{L}^{-1}$ concentration (a) $\Delta \lambda=15 \mathrm{~nm}\left(\lambda_{\mathrm{ex}}=265-305 \mathrm{~nm}\right)$ and $(\mathbf{b}) \Delta \lambda=60 \mathrm{~nm}\left(\lambda_{\mathrm{ex}}=220-440 \mathrm{~nm}\right)$. Insert: comparison of (af)gHSA and (af)gHSA phys emission fluorescence spectra excited at (a) $\lambda_{\mathrm{ex}}=275 \mathrm{~nm}$ and (b) $\lambda_{\mathrm{ex}}=295 \mathrm{~nm} ; \mathrm{t}=37^{\circ} \mathrm{C}$. 


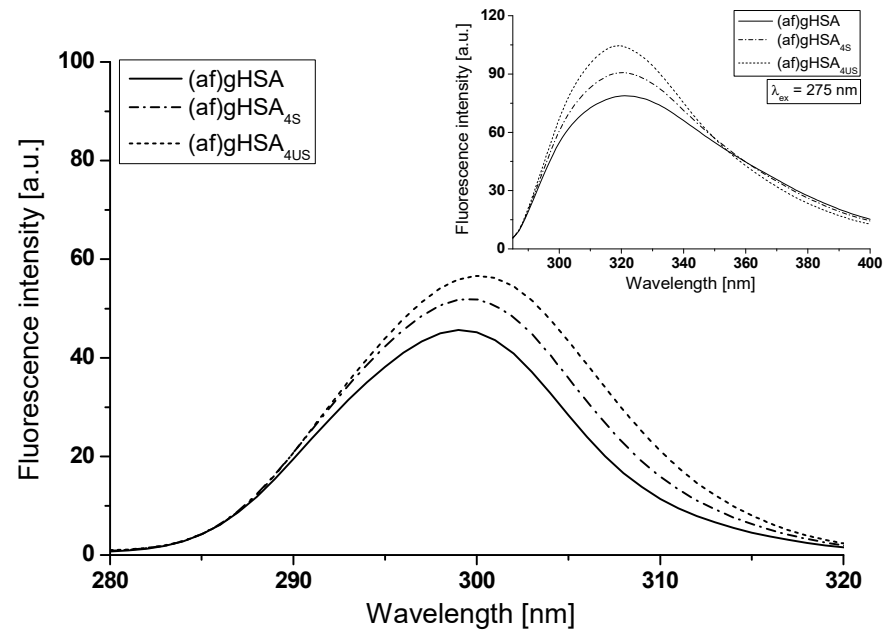

(a)

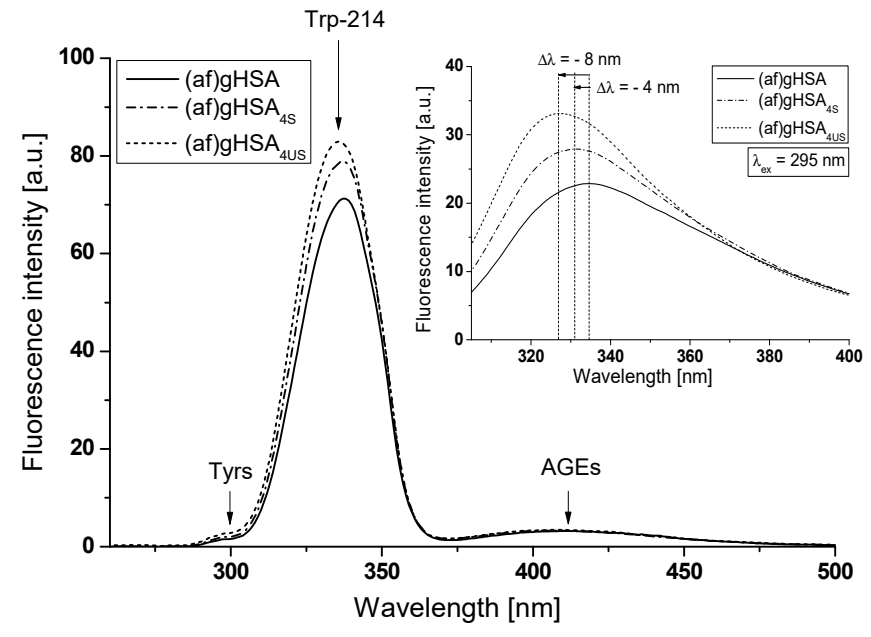

(b)

Figure 11. Main view: synchronous fluorescence spectra of (af)gHSA, (af)gHSA 4 , (af)gHSA 4 Us at $5 \times 10^{-6} \mathrm{~mol} \cdot \mathrm{L}^{-1}$ concentration (a) $\Delta \lambda=15 \mathrm{~nm}\left(\lambda_{\mathrm{ex}}=265-305 \mathrm{~nm}\right)$ and $(\mathbf{b}) \Delta \lambda=60 \mathrm{~nm}\left(\lambda_{\mathrm{ex}}=220-440 \mathrm{~nm}\right)$. Insert: comparison of (af)gHSA, (af)gHSA 4 , (af)gHSA 4 Us emission fluorescence spectra excited at (a) $\lambda_{\mathrm{ex}}=275 \mathrm{~nm}$ and (b) $\lambda_{\mathrm{ex}}=295 \mathrm{~nm} ; \mathrm{t}=37^{\circ} \mathrm{C}$.

Synchronous fluorescence spectroscopy allows for separation of the emission spectra originating from the Trp-214 and Tyrs (as illustrated in Figures 10, 11 and 12b, main view), which results more specific information about the structure of the macromolecules. According to literature data $[25,28,32]$, the synchronous fluorescence spectra were obtained considering the wavelength intervals $\Delta \lambda=60 \mathrm{~nm}$ and $\Delta \lambda=15 \mathrm{~nm}$ to evidence the Trp-214 and Tyrs, respectively $\left(\Delta \lambda=\lambda_{\mathrm{em}}-\lambda_{\mathrm{ex}}\right)$.

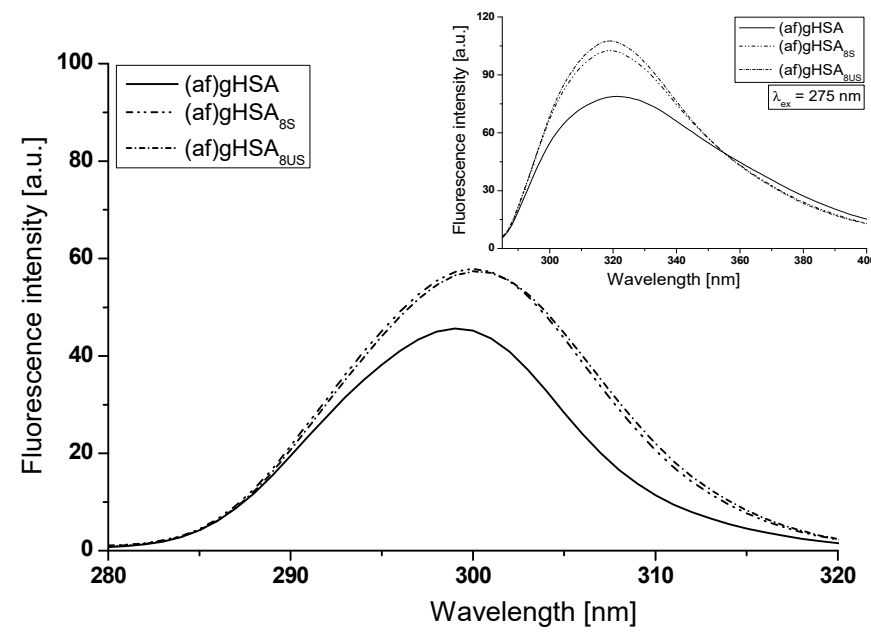

(a)

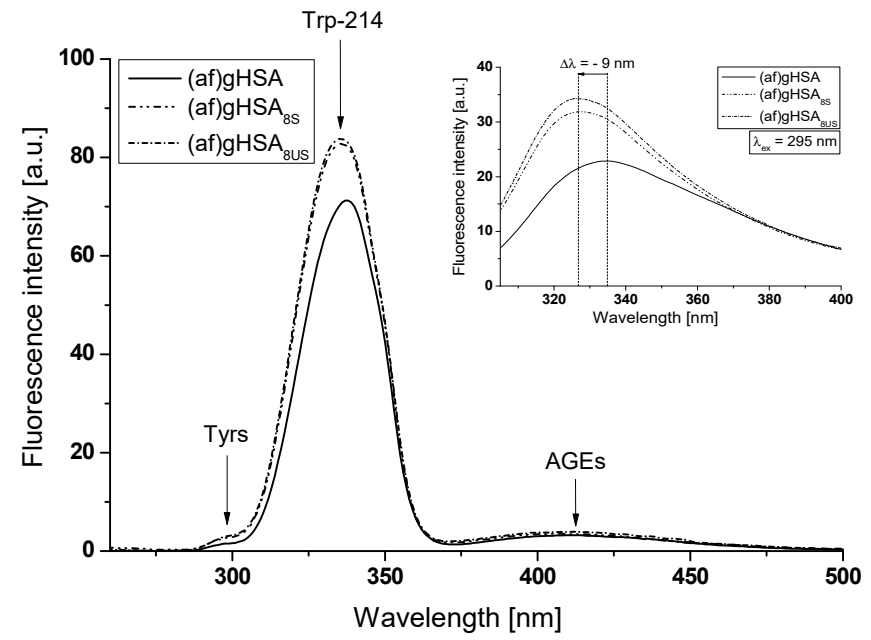

(b)

Figure 12. Main view: synchronous fluorescence spectra of (af)gHSA, (af)gHSA 8 , (af)gHSA 8 Us at $5 \times 10^{-6} \mathrm{~mol} \cdot \mathrm{L}^{-1}$ concentration (a) $\Delta \lambda=15 \mathrm{~nm}\left(\lambda_{\text {ex }}=265-305 \mathrm{~nm}\right)$ and (b) $\Delta \lambda=60 \mathrm{~nm}\left(\lambda_{\text {ex }}=220-440 \mathrm{~nm}\right)$. Insert: comparison of (af)gHSA, (af)gHSA 8 , (af)gHSA 8 Us emission fluorescence spectra excited at (a) $\lambda_{\mathrm{ex}}=275 \mathrm{~nm}$ and (b) $\lambda_{\mathrm{ex}}=295 \mathrm{~nm} ; \mathrm{t}=37^{\circ} \mathrm{C}$.

The fluorescence of human serum albumin fluorophores is sensitive to the changes of HSA tertiary structure and environmental properties. Slight structural changes in albumin near the Trp-214 and Tyrs residues affect the fluorescence intensity $\left(\mathrm{F}_{\max }\right)$ and position of maximum fluorescence ( $\lambda_{\max }$ ) [33]. A blue shift of $\lambda_{\max }$ indicates that the Trp-214 and Tyrs residues are located in a more hydrophobic environment, while a red-shift of $\lambda_{\max }$ 
implies that the amino acid residues are in a polar environment and are more exposed to the solvent [34].

Using $\Delta \lambda=15 \mathrm{~nm}$ (Figures 10a, 11 and 12a, main view) and $\Delta \lambda=60 \mathrm{~nm}$ (Figures 10, 11 and $12 \mathrm{~b}$, main view) no changes were observed in the maximum emission wavelength of

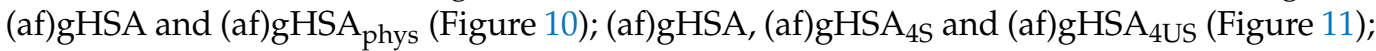
(af)gHSA, (af)gHSA 8 , and (af)gHSA 8 us (Figure 12) Tyrs and Trp-214 residues (Table 4). This indicates the stability of both bands in the synchronous spectra of glycated human serum albumin in the absence (af)gHSA and in the presence of fatty acids (af)gHSA phys, (af)gHSA 4 , (af)gHSA 8 , (af)gHSA 445 , and (af)gHSA 8 us.

Table 4. Intensity of $5 \times 10^{-6} \mathrm{~mol} \cdot \mathrm{L}^{-1}$ (af)gHSA, (af)gHSA phys, (af)gHSA $4 \mathrm{~s}$, (af)gHSA 4 US, (af)gHSA ${ }_{8 \mathrm{~s}}$, and (af)gHSA 8 US emission $\left(\lambda_{\mathrm{ex}}=275 \mathrm{~nm}, \lambda_{\mathrm{ex}}=295 \mathrm{~nm}\right)$ and synchronous $(\Delta \lambda=15 \mathrm{~nm}$ and $\Delta \lambda=60 \mathrm{~nm}$ ) fluorescence spectra.

\begin{tabular}{|c|c|c|c|c|c|c|c|c|}
\hline \multirow{2}{*}{$\begin{array}{l}5 \times 10^{-6} \\
\mathrm{~mol} \cdot \mathrm{L}^{-1}\end{array}$} & \multicolumn{2}{|c|}{$\lambda_{\mathrm{ex}}=275 \mathrm{~nm}$} & \multicolumn{2}{|c|}{$\lambda_{\mathrm{ex}}=295 \mathrm{~nm}$} & \multicolumn{2}{|c|}{$\Delta \lambda=15 \mathrm{~nm}$} & \multicolumn{2}{|c|}{$\Delta \lambda=60 \mathrm{~nm}$} \\
\hline & $\lambda_{\max }(\mathrm{nm})$ & $F_{\max }$ & $\lambda_{\max }(\mathrm{nm})$ & $F_{\max }$ & $\lambda_{\max }(\mathrm{nm})$ & $F_{\max }$ & $\lambda_{\max }(\mathrm{nm})$ & $F_{\max }$ \\
\hline (af)gHSA & 321 & 78.83 & 335 & 22.86 & 299 & 45.62 & 337 & 71.22 \\
\hline (af)gHSA $\mathrm{phys}_{\text {ph }}$ & 320 & 89.78 & 332 & 27.72 & 300 & 51.75 & 337 & 78.40 \\
\hline (af)gHSA 4 S & 321 & 90.73 & 331 & 27.93 & 300 & 51.88 & 337 & 78.91 \\
\hline (af)gHSA $8 \mathrm{~S}$ & 319 & 102.64 & 326 & 31.85 & 300 & 57.90 & 336 & 82.85 \\
\hline (af)gHSA 4 US & 319 & 104.57 & 327 & 33.12 & 300 & 56.68 & 336 & 82.90 \\
\hline (af)gHSA & 319 & 107.61 & 326 & 34.27 & 300 & 57.39 & 336 & 83.84 \\
\hline
\end{tabular}

A lack of synchronous spectra shift (af)gHSA caused by the presence of fatty acids indicates no change in the polarity around Trp-214 and Tyr residues or/and a modification of the structure of glycated human serum albumin in the environment of other residues. On the other hand, the main characteristic of fatted human serum albumin emission fluorescence spectra excited at $\lambda_{\mathrm{ex}}=295 \mathrm{~nm}$ (Figures 10, 11 and 12b, insert) is the blue shift maximum fluorescence for (af)gHSA phys $(\Delta \lambda=3 \mathrm{~nm}$, Figure 10b), for (af)gHSA $4 \mathrm{~S}$ $(\Delta \lambda=4 \mathrm{~nm}$, Figure $11 \mathrm{~b})$, for (af)gHSA $4 \mathrm{US}(\Delta \lambda=8 \mathrm{~nm}$, Figure $11 \mathrm{~b})$, and for (af)gHSA $8 \mathrm{~s}$ and (af)gHSA 8 Us $(\Delta \lambda=9 \mathrm{~nm}$, Figure $12 \mathrm{~b})$, Table 4 .

This phenomenon suggests that Trp-214 of glycated human serum albumin ((af)gHSA ${ }_{\text {phys }}$ (af)gHSA 4 , (af)gHSA 8 , (af)gHSA 4 US, and (af)gHSA 8 US) in the presence fatty acids are less exposed to the solvent than deffated macromolecule ((af)gHSA). The signal at $\lambda_{\mathrm{em}} \cong 410 \mathrm{~nm}$ obtained in synchronous mode of (af)gHSA, (af)gHSA phys, (af)gHSA 4 , (af)gHSA 4 Us, (af)gHSA ${ }_{8 S}$, and (af)gHSA 8 US (Figures 10-12, insert) indicates the presence of fluorescent AGEs-pentosidines and argyrimidines and or/ additional fluorophores created in albumin glycation [35].

The fluorescence intensity of both types of fluorophores in the (af)gHSA spectrum is lower than in the (af)gHSA ${ }_{\text {phys }}$, (af)gHSA 4 , (af)gHSA 8 , (af)gHSA 4 US, and (af)gHSA 8 US spectra (Table 4 ). These results indicate an alteration of the glycated albumin tertiary structure by binding of fatty acids in the region of tryptophanyl and tyrosyl residues occurrence in subdomain IIA (Trp-214, Tyr-263), IB (Tyr-138, Tyr-140, Tyr-148, Tyr-150, and Tyr-161), IIB (Tyr-319, Tyr-332, Tyr-334, Tyr-341, Tyr-353, and Tyr-370), and IIIA (Tyr-401, Tyr-411, Tyr-452, and Tyr-497).

Red Edge Excitation Shift (REES) is an another method to directly monitor of the region surrounding the tryptophanyl residue of glycated, deffated, and glycated in the presence of fatty acids human serum albumin $[33,36]$. In order to study the REES effect, fluorescence spectra of glycated human serum albumin in the absence (af)gHSA and in the presence of fatty acids (af)gHSA phys (af)gHSA 4 , (af)gHSA 8 , (af)gHSA 4 Us, and (af)gHSA 8 Us excited at $\lambda_{\mathrm{ex}}=290 \mathrm{~nm}, \lambda_{\mathrm{ex}}=295 \mathrm{~nm}$, and $\lambda_{\mathrm{ex}}=300 \mathrm{~nm}$ wavelengths were recorded (data not shown).

Emission fluorescence spectra of (af)gHSA Trp-214 residue are different than for Trp-214 of (af)gHSA phys $_{\text {, (af)gHSA }}$, (af)gHSA 8 , (af)gHSA 4 US, and (af)gHSA 8 us at all excitation wavelengths. A slight red-shift maximum emission fluorescence of (af)gHSA phys $\left(\Delta \lambda_{\mathrm{em}}=2 \mathrm{~nm}\right)$, 
(af)gHSA $4 \mathrm{~S}\left(\Delta \lambda_{\mathrm{em}}=3 \mathrm{~nm}\right),(\mathrm{af}) \mathrm{gHSA}_{8 \mathrm{~S}}\left(\Delta \lambda_{\mathrm{em}}=3 \mathrm{~nm}\right),(\mathrm{af}) \mathrm{gHSA} \mathrm{HS}_{4}\left(\Delta \lambda_{\mathrm{em}}=3 \mathrm{~nm}\right)$, and (af)gHSA 8 US $\left(\Delta \lambda_{\mathrm{em}}=3 \mathrm{~nm}\right)$ relative to (af)gHSA $\left(\Delta \lambda_{\mathrm{em}}=6 \mathrm{~nm}\right)$ has been obtained. Higher shift for deffated, glycated albumin indicates that fatty acids present in the structure of albumin decreases the mobility of Trp-214 inducing changes of the albumin conformation.

Similarly, a larger REES in the case of modified-glycated ( $\mathrm{gHSA} F \mathrm{FC}, \Delta \lambda_{\mathrm{em}}=5 \mathrm{~nm}$ ) vs. non-modified (HSA, $\Delta \lambda_{\mathrm{em}}=2 \mathrm{~nm}$ ) human serum albumin was also observed in our previous study [28]. This suggest structural modifications in the hydrophobic pocket containing the tryptophanyl residue due to the glycation process, which contribute to stiffening of the Trp-214 environment or/and limited access to the polar solvent.

\section{Materials and Methods}

\subsection{Reagents}

Fatty-acid-free human serum albumin ((af)HSA, Lot No. 6312A) was purchased from MP Biomedicals LLC (Inc. Illkirch, France). Myristic acid (MYR, Lot No. R28576), oleic acid (OA, Lot No. 1912J), linoleic acid (LA, Lot No. 2353J), palmitic acid (PA, Lot No. 6798H), and stearic acid (SA, Lot No. 7729H) were provided by MP Biomedicals ${ }^{\mathrm{TM}}$ (OH, USA). Sodium azide $\left(\mathrm{NaN}_{3}\right.$, Lot No. BCBD6941V) and losartan (LOS, Lot No. SLBF5611V) were obtained from Sigma-Aldrich Chemical Co. (Darmstadt, Germany) and Sigma-Aldrich Chemical Co. (Shanghai, China), respectively.

D(+)-glucose (GLC, Lot No. A0299881) was supplied by POCH S.A. (Gliwice, Poland). All chemicals were of the highest analytical quality. The stock solutions of MYR, OA, LA, PA, and SA were prepared by dissolving appropriate amounts in methanol for spectroscopy from Merck KGaA (Darmstadt, Germany, Lot No. 32373611/18).

\subsection{In Vitro Glycation of Defatted Human Serum Albumin}

Prior to in vitro glycation of defatted human serum ((af)HSA) albumin, all glass materials and spatulas were sterilized using a lab dryer at $100{ }^{\circ} \mathrm{C}$ prevent bacterial growth. Defatted human serum albumin ((af)HSA) in the presence of glucose (GLC) at $1.0 \times 10^{-5} \mathrm{~mol} \cdot \mathrm{L}^{-1}$ and $0.05 \mathrm{~mol} \cdot \mathrm{L}^{-1}$ concentrations, respectively, were prepared in phosphate buffer solution $\left(\mathrm{pH}=7.4,0.05 \mathrm{~mol} \cdot \mathrm{L}^{-1}\right)$ in double-distilled water and in the presence of sodium azide $\left(\mathrm{NaN}_{3}\right)\left(0.015 \mathrm{~mol} \cdot \mathrm{L}^{-1}\right)$.

Solutions of protein were passed through a sterile Millex-GP syringe filters with $0.2 \mu \mathrm{m}$ pores and then incubated in sterile closed tubes for a period of 21 days at aconstant temperature of $37^{\circ} \mathrm{C}$. After the incubation period, to remove excess unbound glucose, the solution of glycated human serum albumin (af)gHSA was dialyzed extensively against $0.05 \mathrm{~mol} \cdot \mathrm{L}^{-1}$ phosphate buffer at $\mathrm{pH}=7.4$ for $24 \mathrm{~h}$. The $\mathrm{pH} 7.4 \pm 0.1$ of buffer solution was confirmed by pH meter (FEP20 Metler Toledo). The absorbance of (af)HSA and (af)gHSA at 255 and $280 \mathrm{~nm}$ ratio was less than 0.5 , indicating the purity of albumins.

\subsection{Procedure of Preparation Fatty Acids Solutions}

Stock solutions were made for fatty acids (FA) (oleic acid (OA), palmitic acid (PA), stearic acid (SA), myristic acid (MYR), and linoleic acid (LA)) with a concentration of $1 \times 10^{-3} \mathrm{~mol} \cdot \mathrm{L}^{-1}$ by dissolving the right amount of acid in methanol. In order to study the influence of fatty acids on the binding properties of glycated human serum albumin, five FA mixtures with differing contents were prepared in the phosphate buffer; saturated (FAs) and unsaturated (FA $\mathrm{FS}_{\mathrm{US}}$ ) fatty acids (mix 1-5):

1. Fatty acids physiological mixture at $2.0 \times 10^{-5} \mathrm{~mol} \cdot \mathrm{L}^{-1}$ concentration containing FA mixtures at $6 \times 10^{-6} \mathrm{~mol} \cdot \mathrm{L}^{-1} \mathrm{PA}, 0.5 \times 10^{-6} \mathrm{~mol} \cdot \mathrm{L}^{-1} \mathrm{SA}, 0.5 \times 10^{-6} \mathrm{~mol} \cdot \mathrm{L}^{-1} \mathrm{MYR}$ $8 \times 10^{-6} \mathrm{~mol} \cdot \mathrm{L}^{-1} \mathrm{OA}$, and $5 \times 10^{-6} \mathrm{~mol} \cdot \mathrm{L}^{-1} \mathrm{LA}$ concentrations-mix 1 .

2. Fatty acids compound with a concentration of $4.1 \times 10^{-5} \mathrm{~mol} \cdot \mathrm{L}^{-1}$ with four times the amount of $\mathrm{FA}_{S}$ compared to the physiological mixture containing FA mixtures at $2.4 \times 10^{-5} \mathrm{~mol} \cdot \mathrm{L}^{-1} \mathrm{PA}, 2.0 \times 10^{-6} \mathrm{~mol} \cdot \mathrm{L}^{-1} \mathrm{SA}, 2.0 \times 10^{-6} \mathrm{~mol} \cdot \mathrm{L}^{-1} \mathrm{MYR}, 8.0 \times 10^{-6}$ $\mathrm{mol} \cdot \mathrm{L}^{-1} \mathrm{OA}$, and $5.0 \times 10^{-6} \mathrm{~mol} \cdot \mathrm{L}^{-1} \mathrm{LA}$ concentrations-mix 2 . 
3. A compound of fatty acids with a concentration of $6.9 \times 10^{-5} \mathrm{~mol} \cdot \mathrm{L}^{-1}$ with eight times the amount of $\mathrm{FA}_{\mathrm{S}}$ compared to the physiological mixture containing $4.8 \times 10^{-5} \mathrm{~mol} \cdot \mathrm{L}^{-1} \mathrm{PA}, 4.0 \times 10^{-6} \mathrm{~mol} \cdot \mathrm{L}^{-1} \mathrm{SA}, 4.0 \times 10^{-6} \mathrm{~mol} \cdot \mathrm{L}^{-1} \mathrm{MYR}$, and $8.0 \times 10^{-6} \mathrm{~mol} \cdot \mathrm{L}^{-1} \mathrm{OA}$ and with the concentration of $5.0 \times 10^{-6} \mathrm{~mol} \cdot \mathrm{L}^{-1} \mathrm{LA}$ concentrations-mix 3 .

4. A compound of fatty acids with the concentration of $5.9 \times 10^{-5} \mathrm{~mol} \cdot \mathrm{L}^{-1}$ with four times the amount of $F A_{U S}$ compared to the physiological mixture containing: $6.0 \times 10^{-6} \mathrm{~mol} \cdot \mathrm{L}^{-1} \mathrm{PA}, 5.0 \times 10^{-5} \mathrm{~mol} \cdot \mathrm{L}^{-1} \mathrm{SA}, 5.0 \times 10^{-5} \mathrm{~mol} \cdot \mathrm{L}^{-1} \mathrm{MYR}, 3.2 \times 10^{-5}$ $\mathrm{mol} \cdot \mathrm{L}^{-1} \mathrm{OA}$, and $2.0 \times 10^{-5} \mathrm{~mol} \cdot \mathrm{L}^{-1} \mathrm{LA}-\operatorname{mix} 4$.

5. A compound of fatty acids with the concentration of $1.11 \times 10^{-4} \mathrm{~mol} \cdot \mathrm{L}^{-1}$ with eight times the amount of $\mathrm{FA}_{\mathrm{US}}$ compared to the physiological mixture containing $6.0 \times 10^{-6} \mathrm{~mol} \cdot \mathrm{L}^{-1} \mathrm{PA}, 5.0 \times 10^{-5} \mathrm{~mol} \cdot \mathrm{L}^{-1} \mathrm{SA}, 5.0 \times 10^{-5} \mathrm{~mol} \cdot \mathrm{L}^{-1} \mathrm{MYR}, 6.4 \times 10^{-5}$ $\mathrm{mol} \cdot \mathrm{L}^{-1} \mathrm{OA}$, and $4.0 \times 10^{-5} \mathrm{~mol} \cdot \mathrm{L}^{-1} \mathrm{LA}$ concentrations-mix 5 .

\subsection{Preparation of Samples for Fluorescence and UV-Vis Studies}

Six glycated albumins (af)gHSA samples at $5 \times 10^{-6} \mathrm{~mol} \cdot \mathrm{L}^{-1}$ concentrations were prepared in the absence and in the presence of a suitable fatty acids mixture (albumin

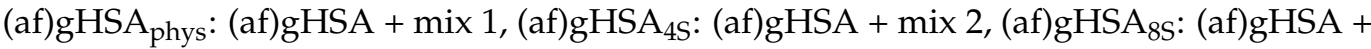
mix 3, (af)gHSA 4 Us: (af)gHSA + mix 4, and (af)gHSA $8 \mathrm{US}$ ): (af)gHSA + mix 5). The content of methanol in the samples did not exceed $1 \%$ of the tested protein solution total volume.

The analysis of the interaction of losartan (LOS) with (af)gHSA, (af)gHSA phys, (af)gHSA (af)gHSA 8 , (af)gHSA 4 Us, and (af)gHSA 8 us was performed using the albumin fluorescence quenching method. Samples for fluorescence and absorbance measurements were made using the titration method. By the use of Hamilton syringe, a suitable volumes of LOS ( $3 \mu \mathrm{L}$ in 10 portions) was added to $3 \mathrm{~mL}$ of albumins immediately before the fluorescence measurement. Due to the equipment limitations, the final molar ratio LOS:(af)gHSA, LOS:(af)gHSA phys $_{1}$ LOS:(af)gHSA 4 , LOS:(af)gHSA 8 , LOS:(af)gHSA 4 Us, and LOS:(af)gHSA 8 Us was 10:1. The stock solution of losartan (LOS) at a $5 \times 10^{-3} \mathrm{~mol} \cdot \mathrm{L}^{-1}$ concentration was prepared in distilled water.

\subsection{Instruments and Measurements Conditions}

The fluorescence and absorbance measurements of the samples were recorded at $37{ }^{\circ} \mathrm{C}$ using a JASCO spectrofluorimeter FP-6500 equipped with a Peltier thermostat $\left(\Delta \mathrm{t} \pm 0.2^{\circ} \mathrm{C}\right.$ ) (error apparatus $\pm 1.5 \mathrm{~nm}$ ) and JASCO spectrophotometer V-760 (the correction of the error of the apparatus for the wavelength and photometric readings was equal to $\pm 0.3 \mathrm{~nm}$ and $\pm 0.002 \mathrm{Abs}$. at $0.5 \mathrm{Abs})$, respectively, and standard quartz cuvettes. The fluorescence spectra presented in the paper were corrected for the phosphate buffer using the Spectra Manager program and then analyzed using OriginPro version 8.5 SR1 software (Northampton, MA, USA). The results of the study were expressed as a mean \pm relative standard deviation (RSD) from three independent experiments.

The emission fluorescence spectra of tryptophanyl (Trp-214) and thyrosyl (Tyr) residues of the proteins ((af)gHSA, (af)gHSA ${ }_{\text {phys }}$ (af)gHSA 4 , (af)gHSA 8 , (af)gHSA 4 Us, and (af)gHSA 8 Us) were recorded at the excitation wavelength $\lambda_{\mathrm{ex}}=275 \mathrm{~nm}\left(\lambda_{\mathrm{em}}=285-400 \mathrm{~nm}\right)$, and the fluorescence spectra of the Trp-214 were measured at $\lambda_{\mathrm{ex}}=295 \mathrm{~nm}\left(\lambda_{\mathrm{em}}=305-400 \mathrm{~nm}\right)$. The synchronous fluorescence spectra were obtained considering the wavelength intervals $\Delta \lambda=15 \mathrm{~nm}$ and $\Delta \lambda=60 \mathrm{~nm}$ to evidence the protein fluorophores Tyr residues and Trp-214 residue, respectively $\left(\Delta \lambda\right.$-difference between emission $\left(\lambda_{\mathrm{em}}\right)$ and excitation $\left(\lambda_{\mathrm{ex}}\right)$ wavelength).

The measurements were conducted when the spectral width of the band (for monochromator of excitation and emission radiation) was equal to $3 \mathrm{~nm}$ with a sample scanning speed of $100 \mathrm{~nm} / \mathrm{min}$. The Red Edge Excitation Shift (REES) of (af)gHSA was compared to (af)gHSA phys $_{\text {, (af)gHSA }}$, (af)gHSA 8 , (af)gHSA 4 Us, and (af)gHSA 8 Us) with the use of $\lambda_{\mathrm{ex}}=290 \mathrm{~nm}, \lambda_{\mathrm{ex}}=295 \mathrm{~nm}$, and $\lambda_{\mathrm{ex}}=300 \mathrm{~nm}$. The measurement ranges of all emission spectra were recorded from 310 to $400 \mathrm{~nm}$ and the slit widths were $3 \mathrm{~nm} / 3 \mathrm{~nm}$. 
The degree of macromolecule fluorescence quenching by the LOS was determined relative to the fluorescence of the non-ligand albumin solutions. Due to the inner filter effect (IFE) caused by the presence of the drug, the recorded fluorescence was corrected using the following formula (Equation (1)) [19]:

$$
\mathrm{F}_{\mathrm{cor}}=\mathrm{F}_{\mathrm{obs}} \cdot 10^{\left(\frac{\mathrm{A}_{\mathrm{ex}}+\mathrm{Aem}}{2}\right)}
$$

where $\mathrm{F}_{\text {cor }}$ and $\mathrm{F}_{\mathrm{obs}}$ are the corrected and observed fluorescence intensity, respectively; $\mathrm{A}_{\mathrm{ex}}$ and $\mathrm{A}_{\mathrm{em}}$ are the absorbance at excitation $\left(\lambda_{\mathrm{ex}}=275 \mathrm{~nm}\right.$ or $\left.\lambda_{\mathrm{ex}}=295 \mathrm{~nm}\right)$ and emission wavelength for (af)gHSA, (af)gHSA ${ }_{\text {phys, }}$ (af)gHSA 4 , (af)gHSA 8 , (af)gHSA 4 Us, and (af)gHSA $8 \mathrm{Us}$, respectively.

\subsection{Analysis of Fluorescence Spectra-Calculation of the Stern-Volmer and Association Constants}

Based on the calculated fluorescence emission intensities in the absence and in the presence of fatty acids glycated human serum albumin, curves of (af)gHSA, (af)gHSA phys, (af)gHSA ${ }_{45}$, (af)gHSA 8 , (af)gHSA 4 Us, and (af)gHSA 8 us fluorescence quenching by losartan (LOS) $\left(\frac{\mathrm{F}}{\mathrm{F}_{0}}\right.$ vs. ligand:albumin molar ratio, where $\mathrm{F}$ and $\mathrm{F}_{0}$ is the fluorescence intensity at the maximum wavelength of albumin in the presence and absence of a quencher, respectively) were drawn.

The quenching effect (static and/or dynamic) fluorescence of (af)gHSA, (af)gHSA phys, (af)gHSA 4 , (af)gHSA 8 , (af)gHSA 4 US, and (af)gHSA 8 US, the Stern-Volmer constants $\mathrm{K}_{\mathrm{SV}}$, the bimolecular quenching rate constants $\mathrm{k}_{\mathrm{q}}\left(\mathrm{k}_{\mathrm{q}}=\mathrm{K}_{\mathrm{SV}} / \tau_{0}\right)$, and maximum available fluorescence fraction of all albumin $\mathrm{f}_{\mathrm{a}}$ fluorophores were analyzed on the basis of the Stern-Volmer equation (Equation (2)) [20]:

$$
\frac{\mathrm{F}_{0}}{\mathrm{~F}}=1+\mathrm{K}_{\mathrm{SV}} \cdot[\mathrm{L}]=1+\mathrm{k}_{\mathrm{q}} \tau_{0} \cdot[\mathrm{L}]
$$

where $\mathrm{k}_{\mathrm{q}}$ is the bimolecular quenching rate constant $\left[\mathrm{mol}^{-1} \cdot \mathrm{L} \cdot \mathrm{s}^{-1}\right] ; \tau_{0}$ is the average fluorescence lifetime of albumin without of quencher $\tau_{0}=6.0 \times 10^{-9} \mathrm{~s}$ [22]; $\mathrm{K}_{\mathrm{SV}}$ is the Stern-Volmer constant $\left[\mathrm{mol}^{-1} \cdot \mathrm{L}\right]$; and $[\mathrm{L}]$ is the ligand concentration $\left[\mathrm{mol} \cdot \mathrm{L}^{-1}\right.$ ] where $[\mathrm{L}]=\left[\mathrm{L}_{\mathrm{b}}\right]+\left[\mathrm{L}_{\mathrm{f}}\right],\left[\mathrm{L}_{\mathrm{b}}\right]$ and $\left[\mathrm{L}_{\mathrm{f}}\right]$ are the bound and free (unbound) drug concentrations $\left[\mathrm{mol} \cdot \mathrm{L}^{-1}\right]$.

The quenching parameters $\left(\mathrm{K}_{\mathrm{SV}}, \mathrm{k}_{\mathrm{q}}\right)$ and $\mathrm{f}_{\mathrm{a}}$ for a system with non-linear SternVolmer relationship were calculated using the Stern-Volmer equation modified by Lehrer (Equation (3)) [21]:

$$
\frac{\mathrm{F}_{0}}{\Delta \mathrm{F}}=\frac{1}{[\mathrm{~L}]} \cdot \frac{1}{\mathrm{f}_{\mathrm{a}}} \cdot \frac{1}{\mathrm{~K}_{\mathrm{SV}}}+\frac{1}{\mathrm{f}_{\mathrm{a}}}
$$

where $f_{a}$ is the fractional maximum protein fluorescence accessible for the quencher.

Isotherms of losartan binding to glycated human serum albumin in the absence and in the presence of fatty acids were obtained based on the graph of the function $r=f\left(\left[\mathrm{~L}_{\mathrm{f}}\right]\right)$, where $\mathrm{r}=\frac{\left[\mathrm{L}_{\mathrm{b}}\right]}{[(\mathrm{af}) \mathrm{gHSA}]}$ is the number of ligands moles bound per mole of protein molecule; $\left[\mathrm{L}_{\mathrm{b}}\right]=\frac{\Delta \mathrm{F}}{\Delta \mathrm{F}_{\max }} \times(\mathrm{af}) \mathrm{gHSA}_{\text {total }}, \Delta \mathrm{F}$ is the difference between $\mathrm{F}_{0}$ and $\mathrm{F} ; \Delta \mathrm{F}_{\max }$ (the maximal fluorescence change with complete saturation) is evaluated from the linear part of the $\frac{1}{\Delta \mathrm{F}} \mathrm{Vs}$. $\frac{1}{[\mathrm{~L}]} ;$ and $[(\mathrm{af}) \mathrm{gHSA}]$ is serum albumin concentration $\left[\mathrm{mol} \cdot \mathrm{L}^{-1}\right][29]$.

From the Scatchard (Equation (4)) [37] and the Klotz (Equation (5)) [38] curves, the values of $\mathrm{K}_{\mathrm{a}}$ association constants and $n$ the number of binding sites in the albumin molecule were determined.

$$
\begin{aligned}
& \frac{\mathrm{r}}{\left[\mathrm{L}_{\mathrm{f}}\right]}=n \cdot \mathrm{K}_{\mathrm{a}}-\mathrm{K}_{\mathrm{a}} \cdot \mathrm{r} \\
& \frac{1}{\mathrm{r}}=\frac{1}{n}+\frac{1}{n \cdot \mathrm{K}_{\mathrm{a}} \cdot\left[\mathrm{L}_{\mathrm{f}}\right]}
\end{aligned}
$$


Hill's coefficient $\mathrm{n}_{\mathrm{H}}$ was determined on the basis of the Hill method (Equation (6)) [39]:

$$
\log \left(\frac{\mathrm{r}}{1-\mathrm{r}}\right)=\mathrm{n}_{\mathrm{H}} \cdot \log \left[\mathrm{L}_{\mathrm{f}}\right]+\log \mathrm{K}_{\mathrm{a}}
$$

\section{Conclusions}

Fluorescence spectroscopic measurements confirmed that losartan (LOS) interacts with glycated human serum albumin (af)gHSA, both in the absence and in the presence of fatty acids ((af)gHSA phys (af)gHSA 4 , (af)gHSA 8 , (af)gHSA 4 Us, and (af)gHSA 8 Us) and quenches albumin fluorescence intensity via a static quenching mechanism. Based on the obtained data, we can conclude that LOS molecules locate closer to the fluorophores of glycated fatted by fatty acids physiological mixture albumin (af)gHSA phys $_{\text {compared with }}$ glycated defatted albumin (af)gHSA fluorophores.

They also locate at different distances to fluorophores of glycated albumin containing various amounts of saturated and unsaturated fatty acids. Moreover, LOS binds not only to its specific binding sites in albumins but also non-specifically interacts with the hydrophobic fragments of its surface and has the same affinity for (af)gHSA and (af)gHSA phys $_{\text {albumin }}$ binding sites. A higher concentration of both saturated and unsaturated fatty acids in glycated albumin reduces the stability of the complex formed with the drug.

According to our results, we can also conclude that, in the case of drugs with a high degree of protein binding (such as losartan) or with a narrow therapeutic index, no changes in the concentration of the albumin in the blood result in a change in the drug's free fraction concentration responsible for therapeutic effects. The presented study allowed for determination of the binding capacity of albumin that is structurally changed by glycation and/or in the presence of fatty acids. This can be used to model drug-protein interactions simulating pathological conditions in the body.

Author Contributions: A.S. conceived and designed the experiments, performed the research, analyzed and discussed the data, and wrote the paper; J.P. conducted critical revision and analyzed the data; K.K., A.O. and W.R. contributed to analyzing the data; and M.M.-J. translated the text and analyzed the data. All authors have read and agreed to the published version of the manuscript.

Funding: This research was funded by Grant PCN-2-105/K/0/F from the Medical University of Silesia, Poland.

Institutional Review Board Statement: Not applicable.

Informed Consent Statement: Not applicable.

Data Availability Statement: Not applicable.

Conflicts of Interest: The authors declare no conflict of interest.

Sample Availability: Samples of the compounds are available from the authors.

\section{References}

1. Peters, T. All about Albumin. In Biochemistry, Genetics and Medical Applications; Academic Press: San Diego, CA, USA, 1995; pp. 1-40.

2. Shamsi, A.; Ahmed, A.; Bano, B. Probing the interaction of anticancer drug temsirolimus with human serum albumin: Molecular docking and spectroscopic insight. J. Biomol. Struct. Dyn. 2017, 36, 1479-1489. [CrossRef]

3. Ahmed, A.; Shamsi, A.; Khan, M.S.; Husain, F.M.; Bano, B. Methylglyoxal induced glycation and aggregation of human serum albumin: Biochemical and biophysical approach. Int. J. Biol. Macromol. 2018, 113, 269-276. [CrossRef]

4. Al-Harthia, S.; Lachowicz, J.I.; Nowakowski, M.E.; Jaremko, M.; Jaremko, Ł. Towards the functional high-resolution coordination chemistry of blood plasma human serum albumin. J. Inorg. Biochem. 2019, 198, 110716. [CrossRef]

5. Vlassara, H.; Bucala, R.; Striker, L. Pathogenic effects of advanced glycosylation: Biochemical, biologic, and clinical implications for diabetes and aging. Lab. Investig. 1994, 70, 138-151.

6. Cohen, M.P. Clinical, pathophysiological and structure/function consequences of modification of albumin by Amadori-glucose adducts. Biochim. Biophys. Acta 2013, 1830, 5480-5485. [CrossRef]

7. Berk, P.D.; Stump, D.D. Mechanisms of cellular uptake of long chain free fatty acids. Mol. Cell. Biochem. 1999, 192, 17-31. [CrossRef] 
8. Van der Vusse, G.J. Albumin as fatty acid transporter. Drug Metab. Pharmacokinet. 2009, 24, 300-307. [CrossRef]

9. Sudlow, G.; Birkett, D.J.; Wade, D.N. The characterization of two specific drug binding sites on human serum albumin. Mol. Pharmacol. 1975, 11, 824-832.

10. Simard, J.R.; Zunszain, P.A.; Hamilton, J.A.; Curry, S. Locating of high and low affinity fatty acid binding sites on human serum albumin revealed by NMR drug-competition analysis. J. Mol. Biol. 2006, 361, 336-351. [CrossRef]

11. Ascenzi, P.; Di Masi, A.; Fanali, G.; Fasano, M. Heme-based catalytic properties of human serum albumin. Cell. Death. Dis. 2015, 1, 15025. [CrossRef]

12. Fanali, G.; di Masi, A.; Trezza, V.; Marino, M.; Fasano, M.; Ascenzi, P. Human serum albumin: From bench to bedside. Mol. Asp. Med. 2012, 33, 209-290. [CrossRef]

13. Lee, P.; Wu, X. Review: Modifications of Human Serum Albumin and Their Binding Effect. Cur. Pharm. Des. 2015, 21, 1862-1865. [CrossRef]

14. Bhattacharya, A.A.; Curry, S.; Franks, N.P. Binding of the general anesthetics propofol and halothane to human serum albumin: High resolution crystal structures. J. Biol. Chem. 2000, 275, 38731-38738. [CrossRef]

15. Anguizola, J.A.; Basiaga, S.B.G.; Hage, D.S. Effects of fatty acids and glycation on drug interactions with human serum albumin. Curr. Metab. 2013, 1, 239-250.

16. Czernichow, S.; Kengne, A.P.; Huxley, R.R.; Batty, G.D.; De Galan, B.; Grobbee, D.; Pillai, A.; Zoungas, S.; Marre, M.; Woodward, M.; et al. Comparison of waist-to-hip ratio and other obesity indices as predictors of cardiovascular disease risk in people with type-2 diabetes: A prospective cohort study from ADVANCE. Eur. J. Cardiovasc. Prev. Rehabil. 2011, 18, 312-319. [CrossRef]

17. Smajić, M.; Vujić, Z.; Mulavdić, N.; Brborić, J. An Improved HPLC Method for Simultaneous Analysis of Losartan Potassium and Hydrochlorothiazide with the Aid of a Chemometric Protocol. Chromatographia 2013, 76, 419-425. [CrossRef]

18. Stryer, L. Fluorescence spectroscopy of proteins. Science 1978, 162, 526-533. [CrossRef]

19. Lakowicz, J.R. Principles of Fluorescence Spectroscopy, 3rd ed.; Springer: New York, NY, USA, 2006; pp. $130-135$.

20. Eftink, M.R.; Ghiron, C.A. Fluorescence quenching studies with proteins. Anal. Biochem. 1981, 114, 199-227. [CrossRef]

21. Lehrer, S.S. Solute Perturbation of Protein Fluorescence. The Quenching of the Tryptophanyl Fluorescence of Model Compounds and of Lysozyme by Iodide Ion. Biochemistry 1971, 10, 3254-3263. [CrossRef]

22. Lakowicz, J.R.; Weber, G. Quenching of Protein Fluorescence by Oxygen. Detection of Structural Fluctuations in Proteins on the Nanosecond Time Scale. Biochemistry 1973, 12, 4171-4179. [CrossRef]

23. Szkudlarek, A.; Wilk, M.; Maciążek-Jurczyk, M. In Vitro Investigations of Acetohexamide Binding to Glycated Serum Albumin in the Presence of Fatty Acid. Molecules 2020, 25, 2340. [CrossRef] [PubMed]

24. Albani, J.R. New insights in the interpretation of tryptophan fluorescence. J. Fluoresc. 2007, 17, 406-417. [CrossRef] [PubMed]

25. Moeinpour, F.; Mohseni-Shahri, F.S.; Malaekeh-Nikouei, B.; Nassirli, H. Investigation into the interaction of losartan with human serum albumin and glycated human serum albumin by spectroscopic and molecular dynamics simulation techniques: A comparison study. Chem.-Biol. Interact. 2016, 257, 4-13. [CrossRef] [PubMed]

26. Valeur, B. Molecular Fluorescence: Principles and Applications; Wiley-VCH: London/Weinheim, UK, 2002.

27. Szkudlarek, A.; Pożycka, J.; Maciążek-Jurczyk, M. Influence of Piracetam on Gliclazide—Glycated Human Serum Albumin Interaction. A Spectrofluorometric Study. Molecules 2019, 24, 111. [CrossRef]

28. Szkudlarek, A.; Pentak, D.; Ploch, A.; Pożycka, J.; Maciążek-Jurczyk, M. In Vitro Investigation of the Interaction of Tolbutamide and Losartan with Human Serum Albumin in Hyperglycemia States. Molecules 2017, 22, 2249. [CrossRef] [PubMed]

29. Taira, Z.; Terada, H. Specific and non-specific ligand binding to serum albumin. Biochem. Pharmacol. 1985, 34, $1999-2005$.

30. Rondeau, P.; Bourdon, E. The glycation of albumin: Structural and functional impacts. Biochimie 2011, 93, 645-658. [CrossRef]

31. Katrahalli, U.; Jaldappagari, S.; Kalanur, S.S. Study of the interaction between fluoxetine hydrochloride and bovine serum albumin in the imitated physiological conditions by multi-spectroscopic methods. J. Lumin. 2010, 130, 211-216. [CrossRef]

32. Maciążek-Jurczyk, M.; Janas, K.; Pożycka, J.; Szkudlarek, A.; Rogóż, W.; Owczarzy, A.; Kulig, K. Human Serum Albumin aggregation/Fibrillation and its Abilities to Drug Binding. Molecules 2020, 25, 618. [CrossRef]

33. Demchenko, A.; Ladokhin, A. Red-edge-excitation fluorescence spectroscopy of indole and tryptophan. Eur. Biophys. J. 1988, 15, 369-379. [CrossRef]

34. Miller, J.N. Recent advances in molecular luminescence analysis. Proc. Anal. Div. Chem. Soc. 1979, 16, $203-208$.

35. Kessel, L.; Kalinin, S.; Nagaraj, R.H.; Larsen, M.; Johansson, L.B. Time-resolved and steady-state fluorescence spectroscopic studies of the human lens with comparison to argpyrimidine, pentosidine and 3-OH-kynurenine. Photochem. Photobiol. 2002, 76, 549-554. [CrossRef]

36. Chattopadhyay, A. Exploring membrane organization and dynamics by the wavelength-selective fluorescence approach. Chem. Phys. Lipids 2003, 122, 3-17. [CrossRef]

37. Hiratsuka, T. Conformational changes in the 23-kilodalton $\mathrm{NH}_{2}$-terminal peptide segment of myosin ATPase associated with ATP hydrolysis. J. Biol. Chem. 1990, 265, 18786-18790. [CrossRef]

38. Klotz, I.M.; Hunston, D.L. Properties of graphical representations of multiple classes of binding sites. Biochemistry 1971, 10, 3065-3069. [CrossRef]

39. Hill, A.V. The possible effects of the aggregation of the molecules of hemoglobin on its dissociation curves. J. Physiol. 1910, 40, 4-7. 